\title{
Spin-resolved thermal signatures of Majorana-Kondo interplay in double quantum dots
}

\author{
Piotr Majek, ${ }^{1, *}$ Krzysztof P. Wójcik, ${ }^{2,3}$ and Ireneusz Weymann ${ }^{1}$ \\ ${ }^{1}$ Institute of Spintronics and Quantum Information, \\ Faculty of Physics, Adam Mickiewicz University in Poznań, \\ ul. Uniwersytetu Poznańskiego 2, 61-614 Poznań, Poland \\ ${ }^{2}$ Institute of Molecular Physics, Polish Academy of Sciences, ul. Smoluchowskiego 17, 60-179 Poznań, Poland \\ ${ }^{3}$ Physikalisches Institut, Universität Bonn, Nussallee 12, D-53115 Bonn, Germany
}

(Dated: February 4, 2022)

\begin{abstract}
We investigate theoretically the thermoelectric transport properties of a T-shaped double quantum dot side-coupled to a topological superconducting nanowire hosting Majorana zero-energy modes. The calculations are performed using the numerical renormalization group method focusing on the transport regime, where the system exhibits the two-stage Kondo effect. It is shown that the leakage of Majorana quasiparticles into the double dot system results in a half-suppression of the second stage of the Kondo effect, which is revealed through fractional values of the charge and heat conductances and gives rise to new resonances in the Seebeck coefficient. The heat conductance is found to satisfy a modified Wiedemann-Franz law. Finally, the interplay of Majorana-induced interference with strong electron correlations is discussed in the behavior of the spin Seebeck effect, a unique feature of the considered setup.
\end{abstract}

\section{INTRODUCTION}

At the time of its publication, Ettore Majorana's rederiving of the Dirac equation in terms of real wave functions [1], the solution of which pointed at a particle that is its own anti-particle, has not attracted much attention. The situation has changed after the Kitaev's proposal to realize Majorana fermions as quasiparticles in quantum wires, and to utilize their non-Abelian statistics in quantum computation protocols [2-6]. The enormous interest in this field stems from the topological protection of Majorana quasiparticles, allowing for fault-tolerant computation [3, 4, 7-10]. It exploded after the first experimental observation of Majorana signatures [11], which followed earlier theoretical proposals $[12,13]$. Since then, fabrication of the so-called Majorana wires has been reported by numerous groups, with still improving quality of the characteristic Majorana features in increasingly sophisticated nanostructures $[10,14,15]$. Up to recently, the Majorana signatures were in fact reduced to detection of zero-bias peaks corresponding to single-electron conductance through the superconducting wire, which is not possible in a trivial Cooper-pair dominated medium. However, this laid ground to a controversy, since such an anomaly can also be a fingerprint of zero-energy Andreev states [16-18] or weak anti-localization effects [19]. With recent interferometric experiments [20, 21], unified theoretical picture [15, 22] and prediction of Majorana oscillations in the microwave spectra as further candidates for signature of the presence of Majorana bound states [23], the controversy may seem close to the conclusion supporting the existence of localized Majorana zero-energy modes (MZMs) at the ends of topological superconduc-

\footnotetext{
*pmajek@amu.edu.pl
}

tor wires, but the discussion among the community continues [24]. Meanwhile, the tremendous improvement of experimental techniques may lead to realization of Majorana modes also in strongly correlated systems involving quantum dots (QDs) [25]. Here, we examine unconventional (magnetic and thermoelectric) signatures of MZM presence in an example of such a device.

Generally, strong electron correlations can lead to various forms of the Kondo effect [26, 27], different types of superconductivity and magnetism, or non-Fermi-liquid phases [27-30], to name just a few. Only very recently have this field reached into the realms of topological materials, such as topological Kondo insulators [31], Weyl-Kondo semi-metals [32], and-most relevant for our study-Kondo-Majorana interplay [33, 34]. In this paper we thus seek for interesting features at the crossroads between strong electron correlations and topologically protected Majorana modes, exploiting a tunable playground of quantum dot systems [25]. It is important to note that there exists a number of works concerning the Majorana-Kondo physics. A study of a nanowire inbetween two leads revealed a fixed point distinct from the conventional Kondo one [35]. In setups with multiple Majorana wires the topological Kondo effect may appear [36], and in some cases the mapping onto multichannel Kondo model is possible [37]. Very recently, a novel correlation-fueled mechanism has been proposed to obtain mobile Majorana modes in exotic two-channel Kondo insulators [38]. Moreover, in various T-shaped configurations, very similar to existing experimental setups for MZM detection [15, 25], perturbative RG analysis indicates yet another distinct fixed point [33], with robust strong-coupling nature and independence of the Kondo temperature $T_{K}$ on the coupling to Majorana wire $V_{M}[33,34]$. Numerical studies confirm the former [39], and even show that $T_{K}$ increases with enhancing the coupling to the Majorana mode in the case of single quan- 


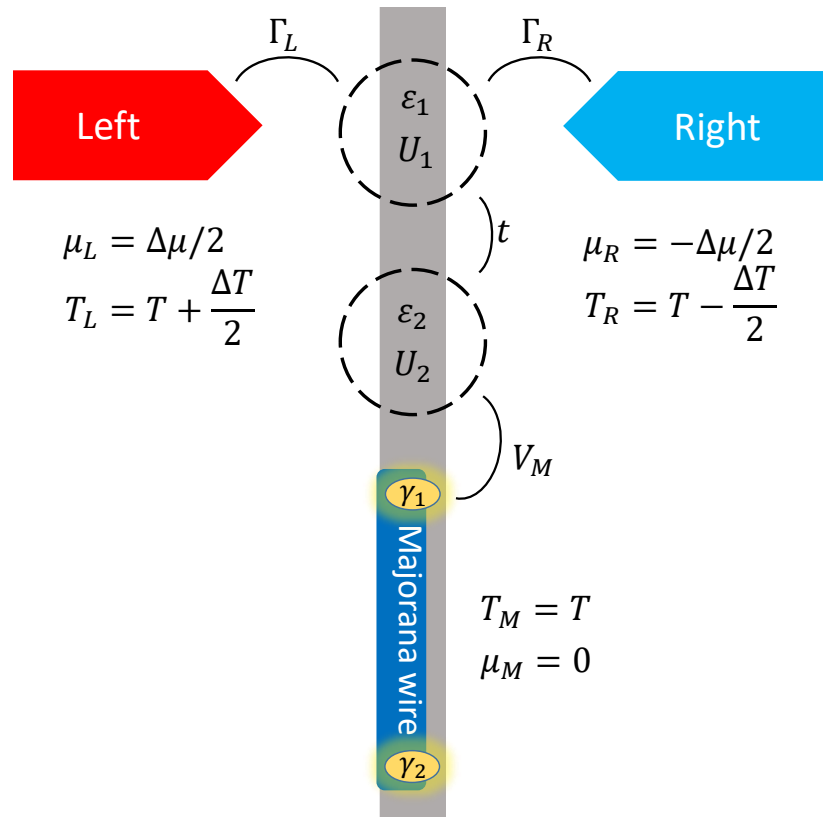

FIG. 1. The schematic of the considered system. The first quantum dot (of on-site energy $\varepsilon_{1}$ and Coulomb correlations $\left.U_{1}\right)$ is connected to metallic leads with the strength $\Gamma_{r}$, with $r=L(R)$, for the left (right) contact. The second quantum dot (of on-site energy $\varepsilon_{2}$ and Coulomb correlations $U_{2}$ ) is coupled to the topological superconducting nanowire, hosting Majorana quasiparticles denoted by $\gamma_{1}$ and $\gamma_{2}$, with coupling matrix elements $V_{M}$. The hopping between the dots is described by $t$. The nanowire temperature is assigned as $T$ and there is a temperature $\Delta T$ and chemical potential $\Delta \mu$ gradient applied between the leads.

tum dots $[40,41]$. In addition to the modification of the relevant energy scales $[25,39,42-44]$, the side-coupling to Majorana wire also gives rise to new fractional values of the conductance. In particular, for single dots a $25 \%$ reduction of the conductance to $3 e^{2} / 2 h$ has been found $[39,41]$. However, in this case the relative change is not large and may be difficult to be detected. Much more interesting in this regard is a double quantum dot (DQD) scenario, where the two-stage Kondo effect develops [45-49], in which at low temperatures the conductance becomes fully suppressed. The presence of Majorana mode results then in an increase of the conductance to some new fractional value [44]. Consequently, the relative change induced by the Majorana mode is much more pronounced in the case of double dots instead of single quantum dot setups. Besides, the considered system allows us to address more fundamental questions regarding the Majorana-Kondo interplay in the presence of more exotic Kondo states. While such questions have already been partly addressed in the case of electronic transport, their thermoelectric signatures remain still rather unexplored. Therefore, the main focus of this paper is on the caloritronic transport properties of a T-shaped double quantum dot side-attached to Majorana wire, as schematically depicted in Fig. 1.
Studying the response of the system subject to thermal gradient gives an important information about the interplay between the Majorana and Kondo physics. Signatures of such interplay have been identified for hybrid devices comprising single quantum dot coupled to metallic leads and to MZM $[50,51]$. In this regard, however, the thermoelectric way of exploring Majorana modes seems particularly unexplored, especially as far as more complex structures are considered. This is despite the fact that thermopower can provide additional insight into the properties of the system, which has been achieved for Kondo QDs both theoretically [52] and experimentally $[53,54]$, and even more could be taken out from the spin-resolved caloritronics $[51,55]$. It has been also predicted that half-fermionic nature of Majorana quasiparticles may give rise to the violation of the WiedemannFranz law [56-58] and it should leave various signatures in QDs weakly coupled with Majorana modes [59-64]. Here, we especially show that a generalized WiedemannFranz law holds for strongly correlated DQD-Majorana system, as in other Kondo scenarios where topology does not play any role $[52,65]$. Furthermore, the presence of the second Kondo scale (introduced by the second QD) in our setup allows for tuning the system into the regime where the Seebeck coefficient changes sign in the presence of even very small coupling to the Majorana mode.

Finally, it should be noted that there exist other considerations on DQD Majorana systems [56, 66, 67], however, these works do not capture the Kondo regime. We believe that with rapid improvements in fabrication techniques [68-71], in particular reducing the necessary magnetic fields [72, 73], construction of such devices would be possible soon, and we hope that our study will stimulate further efforts in this direction.

The paper is organized as follows. In Sec. II we present the model of the studied system, define the thermoelectric coefficients and describe the method used in calculations. To gain an intuitive understanding of the transport behavior, in the next section we discuss the noninteracting case. The fully interacting case is analyzed in great detail in Sec. IV. Finally, the paper is summarized in Sec. V.

\section{THEORETICAL FRAMEWORK}

\section{A. Model}

The schematic of the considered DQD-Majorana setup is presented in Fig. 1. It consists of two single-level quantum dots coupled to each other through the hopping $t$, with one of the dots attached to the external leads. The second dot is directly interacting with superconducting nanowire hosting Majorana zero-energy modes described by the operators $\gamma_{1}$ and $\gamma_{2}$. The system can be modeled by the following Hamiltonian $H=$ $H_{\text {leads }}+H_{\text {tun }}+H_{\mathrm{DD}-\mathrm{Maj}}$. The first term stands for the left and right metallic leads, denoted respectively by 
$r=L$ and $r=R$, for the left and right lead, which are modeled as reservoirs of noninteracting quasiparticles

$$
H_{\text {leads }}=\sum_{r=L, R} \sum_{\mathbf{k} \sigma} \varepsilon_{r \mathbf{k}} c_{r \mathbf{k} \sigma}^{\dagger} c_{r \mathbf{k} \sigma} .
$$

Here, $c_{r \mathbf{k} \sigma}^{\dagger}$ is the creation operator for an electron with spin $\sigma$, momentum $\mathbf{k}$ and the energy $\varepsilon_{r \mathbf{k}}$ in the lead $r$. The second term describes the tunneling processes between the first quantum dot and the leads. It is given by

$$
H_{\mathrm{tun}}=\sum_{r=L, R} \sum_{\mathbf{k} \sigma} v_{r}\left(d_{1 \sigma}^{\dagger} c_{r \mathbf{k} \sigma}+c_{r \mathbf{k} \sigma}^{\dagger} d_{1 \sigma}\right)
$$

with $v_{r}$ denoting momentum-independent tunnel matrix elements. The operator $d_{1 \sigma}^{\dagger}$ creates an electron with spin $\sigma$ in the first quantum dot. The tunnel coupling results in the broadening of the first dot level, which is given by $\Gamma=\Gamma_{L}+\Gamma_{R}$, where $\Gamma_{r}=\pi \rho_{r} v_{r}^{2}$, with $\rho_{r}$ being the density of states of lead $r$. In the following we assume $\Gamma_{L}=\Gamma_{R} \equiv \Gamma / 2$.

The last term of the total Hamiltonian models the subsytem consisting of double quantum dot and Majorana wire, which can be described by the following effective Hamiltonian

$$
\begin{aligned}
H_{\mathrm{DD}-\mathrm{Maj}}= & \sum_{j=1,2} \sum_{\sigma} \varepsilon_{j} d_{j \sigma}^{\dagger} d_{j \sigma}+\sum_{j=1,2} U_{j} d_{j \uparrow}^{\dagger} d_{j \uparrow} d_{j \downarrow}^{\dagger} d_{j \downarrow} \\
& +\sum_{\sigma} t\left(d_{1 \sigma}^{\dagger} d_{2 \sigma}+d_{2 \sigma}^{\dagger} d_{1 \sigma}\right) \\
& +\sqrt{2} V_{M}\left(d_{2 \downarrow}^{\dagger} \gamma_{1}+\gamma_{1} d_{2 \downarrow}\right)
\end{aligned}
$$

where the first three parts model the double quantum dot. $d_{j \sigma}^{\dagger}$ stands for the creation operator for an electron with spin $\sigma$ on the $j$-th dot with the energy $\varepsilon_{j}, U_{j}$ is the corresponding Coulomb correlation energy and the dots are coupled by the hopping matrix element $t$. The second quantum dot is coupled to Majorana wire with tunnel matrix elements given by $V_{M}[39,41,42,74]$. Note that since the bare double dot Hamiltonian $\left(V_{M}=0\right)$ has a full spin symmetry, one can choose the quantization axis in such a way that it coincides with the Majorana mode. Because of that, the Majorana wire couples only to the spin-down electrons on the double dot $[39,42,74]$. The Majorana operators, $\gamma_{1}$ and $\gamma_{2}$, describe Majorana zeroenergy modes at the ends of topological superconducting wire. These operators can be expressed in terms of an auxiliary fermion operator $f$ as $\gamma_{1}=\left(f^{\dagger}+f\right) / \sqrt{2}$ and $\gamma_{2}=i\left(f^{\dagger}-f\right) / \sqrt{2}$. In our considerations we assume that the wire is much longer than the superconducting coherence length, such that the Majorana modes do not overlap [75], unless stated otherwise.

\section{B. Transport coefficients}

In this paper we are interested in the linear-response thermoelectric transport properties of the setup pre- sented in Fig. 1. The temperature and voltage gradients, $\Delta T$ and $\Delta V$, respectively, are symmetrically applied to the left and right contacts, while the topological superconductor is assumed to be grounded and kept at temperature $T$. Under such assumptions, in the linear response regime, the average electric and heat currents flowing between the normal contacts and the wire vanish, provided the system is left-right symmetric [76]. On the other hand, the electric $I$ and heat $I_{h}$ currents flowing between the left and right leads in the linear response regime can be expressed as [77]

$$
\left(\begin{array}{c}
I \\
I_{h}
\end{array}\right)=\sum_{\sigma}\left(\begin{array}{cc}
e^{2} L_{0 \sigma} & -\frac{e}{T} L_{1 \sigma} \\
-e L_{1 \sigma} & \frac{1}{T} L_{2 \sigma}
\end{array}\right)\left(\begin{array}{c}
\Delta V \\
\Delta T
\end{array}\right),
$$

where the functions introduced above, $L_{n \sigma}$, are given by

$$
L_{n \sigma}=-\frac{1}{h} \int \omega^{n} \frac{\partial f(\omega)}{\partial \omega} \mathcal{T}_{\sigma}(\omega) d \omega .
$$

Here, $f(\omega)$ denotes the Fermi-Dirac distribution function for $\Delta \mu=\Delta T=0$ and $\mathcal{T}_{\sigma}(\omega)$ is the transmission coefficient through the double-dot-Majorana setup for spin $\sigma$. For the considered system's geometry, it can be related to the spectral function of the first quantum $\operatorname{dot}, \mathcal{T}_{\sigma}(\omega)=$ $\pi \Gamma A_{\sigma}(\omega)$, with $A_{\sigma}(\omega)=-\frac{1}{\pi} \operatorname{Im} \mathcal{G}_{\sigma}^{\mathrm{R}}(\omega)$, where $\mathcal{G}_{\sigma}^{R}(\omega)$ is the Fourier transform of the retarded Green's function of the first dot, $\mathcal{G}_{\sigma}^{R}(t)=-i \Theta(t)\left\langle\left\{d_{1 \sigma}(t), d_{1 \sigma}^{\dagger}(0)\right\}\right\rangle$.

Because in our considerations it is assumed that the Majorana mode is coupled to the spin-down electrons, it is interesting to investigate the behavior of spin-resolved transport coefficients [48, 78, 79]. The linear conductance, Seebeck coefficient and heat conductance in the spin channel $\sigma$ can be expressed as

$$
\begin{gathered}
G_{\sigma}=\left(\frac{\partial I_{\sigma}}{\partial \Delta V}\right)_{\Delta T=0}=e^{2} L_{0 \sigma}, \\
S_{\sigma}=\frac{1}{G_{\sigma}}\left(\frac{\partial I_{\sigma}}{\partial \Delta T}\right)_{\Delta V=0}=-\frac{1}{e T} \frac{L_{1 \sigma}}{L_{0 \sigma}}, \\
\kappa_{\sigma}=\left(\frac{\partial I_{h \sigma}}{\partial \Delta T}\right)_{I_{\sigma}=0}=\frac{1}{T}\left(L_{2 \sigma}-\frac{L_{1 \sigma}^{2}}{L_{0 \sigma}}\right),
\end{gathered}
$$

where $I_{\sigma}$ and $I_{h \sigma}$ denote the spin-resolved electric and heat currents. Considering both spin channels, the total conductance can be simply calculated with the formula, $G=G_{\uparrow}+G_{\downarrow}$, whereas the total thermopower is defined as [77], $S=-(1 / e T)\left(L_{1} / L_{0}\right)$, where $L_{n}=\sum_{\sigma} L_{n \sigma}$. On the other hand, for the heat conductance one finds, $\kappa=T^{-1}\left(L_{2}-L_{1}^{2} / L_{0}\right)$. We note that in this analysis we only consider the electronic contribution to the heat conductance, with the contribution due to phonons is assumed to be negligible. This is justified in the lowtemperature regime considered here.

As can be seen from the above formulas, the main task is to find the transmission coefficient through the device and determine the integrals $L_{n \sigma}$ in the most accurate way. We achieve this goal by using the numerical renormalization group (NRG) procedure [80-82], which allows 
for construction of full density matrix [83] and calculation of the relevant Green functions directly from their Lehmann representation.

To perform the calculations, it is convenient to reexpress the last term of the double dot-Majorana wire Hamiltonian in terms of an auxiliary fermionic operator $f$. One then finds, $\sqrt{2} V_{M}\left(d_{2 \downarrow}^{\dagger} \gamma_{1}+\gamma_{1} d_{2 \downarrow}\right)=$ $V_{M}\left(d_{2 \downarrow}^{\dagger}-d_{2 \downarrow}\right)\left(f^{\dagger}+f\right)$.

\section{NON-INTERACTING CASE}

To make our theoretical discourse complete, we would like to begin with the description of the non-interacting case. This allows us to obtain analytical formulas and generate exact results that will serve as a starting point for the further analysis when correlations are relevant. In the absence of electron correlations it is possible to get an exact solution for the transmission coefficient by the equation of motion for the retarded Green's function. Moreover, the two spin channels become in practice independent and one can analyze them separately. Even though in realistic case relaxation processes would come into play and blur the picture, we nevertheless find it instructive to perform such an idealized analysis, which serves as a starting point for the discussion of numerical results presented in the next section.

The spin-up transmission $\mathcal{T}_{\uparrow}(\omega)$ is the same as in the model without the Majorana wire and equals

$$
\mathcal{T}_{\uparrow}(\omega)=\frac{\Gamma^{2}}{\Gamma^{2}+\left(\omega-\varepsilon_{1}+\frac{t^{2}}{\omega-\varepsilon_{2}}\right)^{2}} .
$$

We see that it exhibits two peaks reaching $\mathcal{T}_{\uparrow}(\omega)=1$ at zeros of the term in the bracket, and $\mathcal{T}_{\uparrow}(\omega)=0$ at $\omega=\varepsilon_{2}$. The width of the peaks is of the order of $\Gamma$. A representative illustration of these features for $\varepsilon_{1}=\varepsilon_{2}=$ $\varepsilon$ is shown in Fig. 2(a). The maxima of $\mathcal{T}(\omega)$ correspond to single-particle excitations, in this case associated with additional spin-up electron occupying or missing in the double quantum dot.

Whenever the peak of $\mathcal{T}_{\uparrow}(\omega)$ occurs close to $\omega=0$, this mean there are low-energy charge fluctuations, which give rise to good conductance, as illustrated in Fig. 2(b). This also applies to normalized thermal conductance $\kappa / T$. Actually, for a Fermi liquid at low $T$, such that the Sommerfeld expansion is valid, the Wiedemann-Franz (WF) law is fulfilled, i.e. $\mathcal{L}_{0}^{-1} \kappa(T) / T=G(T)$, with $\mathcal{L}_{0} \equiv \pi^{2} / 3$. More generally, it has been observed that some interacting systems exhibit a modified WF law [65], where the shifted and rescaled heat conductance

$$
\widetilde{\kappa}(T) \equiv \kappa(\alpha T) /(\alpha T)
$$

fulfills $\widetilde{\kappa} / \mathcal{L}_{0}=G$ for $\alpha \approx 2$.

In general, narrow peaks of $\mathcal{T}(\omega)$ close to the Fermi level can also lead to large thermopower [84]. Its sign depends on the slope of $\mathcal{T}(\omega)$ and reveals the type of majority charge carriers (electrons or holes). Moreover, the Seebeck coefficient vanishes at the particle-hole symmetry point. For low temperatures, via the Sommerfeld expansion, one finds $S_{\uparrow} \sim T$. On the other hand, at high $T$ from Eq. (7) follows $S_{\uparrow} \sim T^{-1}$. Such behavior can be seen in Fig. 2(c).

Furthermore, the thermopower may also change sign between different transport regimes. This behavior is visualized e.g. by a short-dashed curve in Fig. 2(c) and the corresponding curve in Fig. 2 (a). For $T \lesssim 2 t$ (peaks separation), most of the relevant spectral weight is above $\omega=0$ due to the peak asymmetry, such that electrons overtake holes in transport and, consequently, $S_{\uparrow}>0$. On the contrary, at high $T$, the peak deep below the Fermi energy becomes relevant (i.e. holes can be excited there by thermal fluctuations), which facilitates the hole transport and the sign of $S_{\uparrow}$ is flipped at $T \sim 2 t$.

The results concerning $\mathcal{T}_{\uparrow}(\omega)$ would be also valid in the second spin channel, if not for the coupling to the Majorana mode. From the equation of motion technique, the Green's function in the spin-down channel is conveniently found as a continued fraction,

$$
\begin{aligned}
\mathcal{G}_{\downarrow}(\omega)= & {\left[\omega-\varepsilon_{1}+i \Gamma-t^{2} / A_{1}(\omega)\right]^{-1}, } \\
& A_{1}(\omega)=\omega-\varepsilon_{2}-2 V_{M}^{2} / A_{2}(\omega), \\
& A_{2}(\omega)=\omega-\varepsilon_{M}^{2} / \omega-2 V_{M}^{2} / A_{3}(\omega), \\
& A_{3}(\omega)=\omega+\varepsilon_{2}-t^{2} /\left[\omega+\varepsilon_{1}+i \Gamma\right],
\end{aligned}
$$

in agreement with Ref. [56]. Note that the above formula is quite general and it also takes into account a finite overlap between Majorana modes [75], which can be included in the effective Hamiltonian via the term $i \varepsilon_{M} \gamma_{1} \gamma_{2}$. This overlap is determined by the ratio of the superconducting coherence length to the length of the wire: if the coherence length is much shorter than the wire, the Majorana modes do not overlap and, consequently, $\varepsilon_{M}=0$. Although the expression for the transmission coefficient is quite cumbersome, it follows from Eq. (11) via simple algebra and one can give the exact formulas for $\mathcal{T}_{\downarrow}(\omega)$ and its derivative at $\omega=0$,

$$
\begin{aligned}
& \mathcal{T}_{\downarrow}(0)=\left\{\begin{array}{ll}
{\left[1+\left(\varepsilon_{1}-t^{2} / \varepsilon_{2}\right)^{2} / \Gamma_{\downarrow}^{2}\right]^{-1}} & \text { for } \varepsilon_{M} \neq 0 \\
\frac{1}{2} & \text { for } \varepsilon_{M}=0
\end{array},\right. \\
& \mathcal{T}_{\downarrow}^{\prime}(0)=\left\{\begin{array}{ll}
\frac{\left(\varepsilon_{1}-t^{2} / \varepsilon_{2}\right)\left[V_{M}^{2} t^{2}+2 \varepsilon_{M}^{2}\left(t^{2}+\varepsilon_{2}^{2}\right)\right]}{\Gamma_{\downarrow}^{2} \varepsilon_{2}^{2} \varepsilon_{M}^{2}\left[1+\left(\varepsilon_{1}-t^{2} / \varepsilon_{2}\right)^{2} / \Gamma_{\downarrow}^{2}\right]^{2}} & \text { for } \varepsilon_{M} \neq 0 \\
\frac{\varepsilon_{2}}{4 V_{M}^{2}}-\frac{\varepsilon_{1} \varepsilon_{2}^{2}}{4 V_{M}^{2} t^{2}}-\frac{\varepsilon_{1}}{t^{2}} & \text { for } \varepsilon_{M}=0
\end{array} .\right.
\end{aligned}
$$

Quite strikingly, for $\varepsilon_{M}=0$ the transmission becomes completely universal at low energies, $\mathcal{T}_{\downarrow}(0)=1 / 2[44,56]$, which is a clear manifestation of half-fermionic nature of Majorana quasiparticles. Moreover, $\mathcal{T}^{\prime}(0)$ becomes independent of $\Gamma$, as the width of the spectral features close to $\omega=0$ is set by the strength of the coupling to the Majorana mode, $V_{M}$. It seems noteworthy that $\mathcal{T}_{\downarrow}\left(\varepsilon_{M}\right)=1 / 2+\mathcal{O}\left(\varepsilon_{M}\right)$, so even though $\mathcal{T}_{\downarrow}(0)$ is not universal for finite $\varepsilon_{M}$, at non-zero temperatures $T \sim \varepsilon_{M}$ 

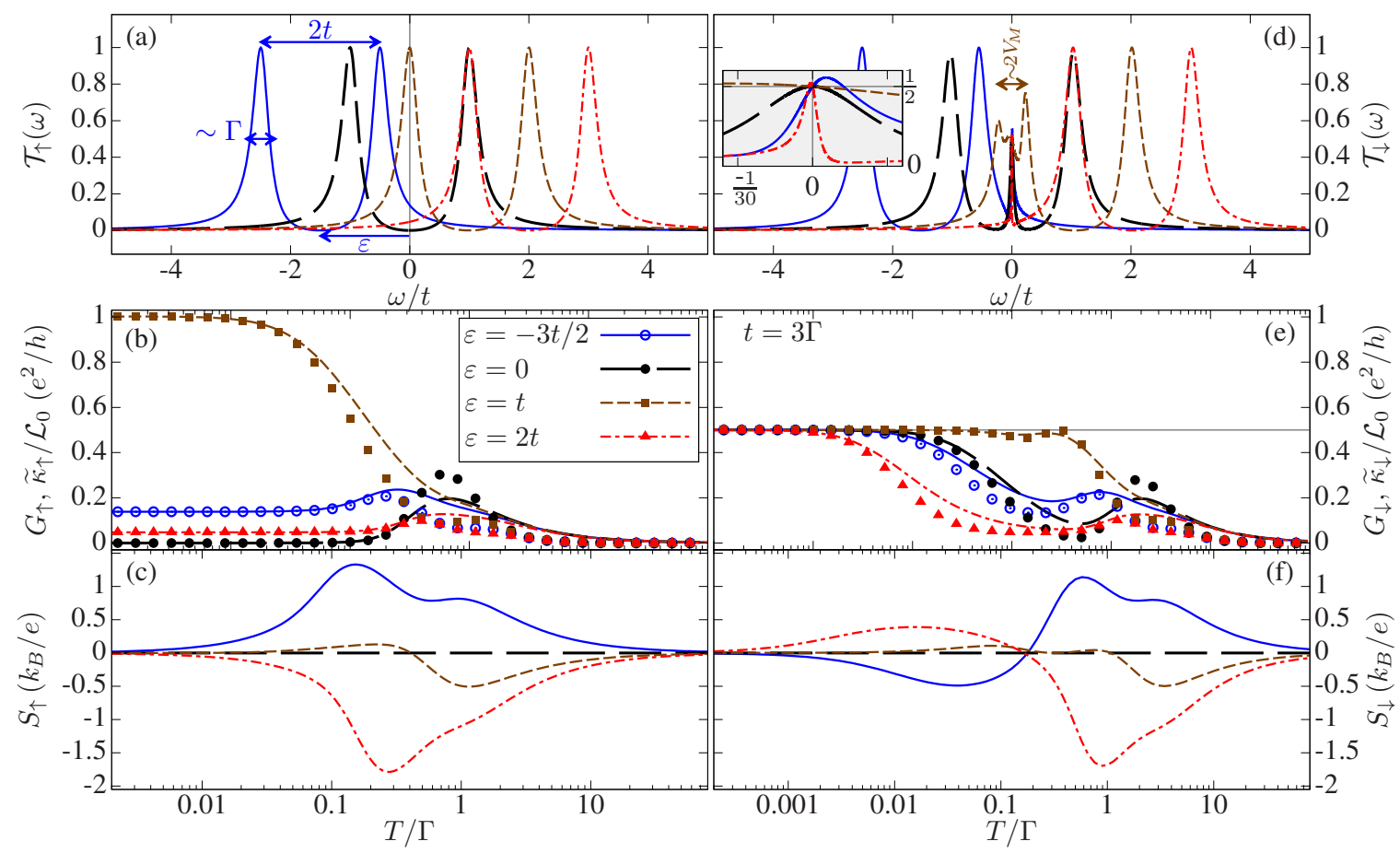

FIG. 2. Left column: (a) $T=0$ transmission coefficient $\mathcal{T}_{\uparrow}(\omega)$ for $\varepsilon=\varepsilon_{1}=\varepsilon_{2}$ indicated in the legend and $t=3 \Gamma$, (b) the conductance $G_{\uparrow}$ as a function of temperature $T$ (lines) and the shifted thermal conductance $\widetilde{\kappa}_{\uparrow}$ (points, see text for details), and (c) spin-up thermopower $S_{\uparrow}$. The right column shows the corresponding plots for the spin-down component. The interaction with Majorana wire is assumed to be $V_{M}=\Gamma / 2$. The inset in (d) zooms the $\omega \approx 0$ region.

one should expect results obtained for $T=\varepsilon_{M}=0$ to be relevant. From now on, let us focus on the $\varepsilon_{M}=0$ case, i.e. long Majorana wire case.

The features discussed above for the spin-down component are visible in Fig. 2(d), and especially in the inset there. However, as is clear from the main plot, far from the Fermi level, the behavior of $\mathcal{T}_{\downarrow}(\omega)$ very much resembles that of $\mathcal{T}_{\uparrow}(\omega)$, compare Fig. 2(a) with Fig. 2(d). Whenever $\mathcal{T}_{\downarrow}(\omega)$ has no peak close to $\omega=0$, just an additional (in general asymmetric) peak appears, such that $\mathcal{T}_{\downarrow}(0)=1 / 2$. Yet the peak of $\mathcal{T}_{\uparrow}(\omega)$ at $\omega=0$ visible in Fig. 2(a) for a short-dashed curve splits into three peaks visible in $\mathcal{T}_{\downarrow}(\omega)$, separated approximately by $V_{M}$. The peaks at $\omega \approx \pm V_{M}$ somewhat resemble the case one would get if Majorana wire was replaced by usual resonant level, while the third peak remains a unique Majorana signature.

The properties of $\mathcal{T}_{\downarrow}(\omega)$ reveal in the corresponding transport characteristics of the system. $G_{\downarrow}(T \rightarrow 0)=e^{2} / 2 h$, irrespective of $\varepsilon$, as can be seen in Fig. 2(e), where the low temperature limit is reached at $T \sim \Gamma_{M} \approx V_{M}^{2} / t$. The modified WF law is still well satisfied at low temperatures, compare the curves and points in Fig. 2(e). On the other hand, the Seebeck coefficient $S_{\downarrow}(T)$ exhibits an additional sign change for $\varepsilon$ away from the resonance, what cannot be seen for the spin-up component of the thermopower, cf. Figs. 2(c) and (f). This sign change occurs for $T / \Gamma \approx 0.1$, and can be related to the corresponding change of slope of the linear conductance, as described by the Mott's formula

$$
S \approx-\frac{\pi^{2}}{3} \frac{k_{B}^{2}}{e} \frac{T}{\mathcal{T}(0)} \frac{\partial \mathcal{T}(\omega)}{\partial \omega} .
$$

\section{NUMERICAL RESULTS AND DISCUSSION}

The considered system reveals interesting effects associated with the interplay between the Kondo and Majorana physics, as compared to bare double quantum dot. In the following sections we present and discuss the numerical results obtained with the aid of the numerical renormalization group method for the fully interacting case. At the beginning, we analyze the spin-resolved charge and thermal conductance in the context of the Wiedemann-Franz law and its persistence in the presence of topological superconductor. We also examine the Majorana-induced current spin polarization. Then, we study the thermopower $S$, where Majorana-induced sign change is observed. In the discussion we focus on the two regimes of hopping between quantum dots: the weak and strong ones. The weak interdot hopping regime is characteristic of the two-stage Kondo effect, whereas when the hopping is strong, a molecular singlet state forms between the dots and the Kondo correlations are less important. 
In NRG computations we take the discretization parameter $\Lambda=2-2.5$ and keep at least 3000 states during the procedure of iterative diagonalization. All energies are expressed in terms of band halfwidth $D$, which is used as energy unit $D \equiv 1$. We set the Coulomb interaction $U_{1}=U_{2} \equiv U=0.2$, while the first quantum dot is coupled to metallic leads with strength $\Gamma_{L}=\Gamma_{R} \equiv$ $\Gamma=U / 10$. Unless otherwise stated, the energy levels of quantum dots are set as $\varepsilon_{1}=\varepsilon_{2} \equiv \varepsilon=-U / 3$. We note that although we focus on the case of symmetric dots, the results presented here are also valid for slightly asymmetric systems, i.e. dots having different charging energies, couplings to the contacts and detuned levels, as long as the system is in the Kondo regime.

\section{A. Persistence of the modified Wiedemann-Franz law}

To begin with, in Figs. 3 and 4 we present the spinresolved and total linear conductance as a function of temperature, calculated for different values of the coupling to the Majorana zero mode in the case of weak (Fig. 3) and strong (Fig. 4) hopping $t$ between the quantum dots. For the weak value of hopping, lack of the coupling to superconducting nanowire results in the typical two-stage Kondo behavior, where besides the Kondo temperature $T_{K}$, defined as [85]

$$
T_{K}=\sqrt{\frac{\Gamma U}{2}} \exp \left[\frac{\pi}{2} \frac{\varepsilon_{1}\left(\varepsilon_{1}+U\right)}{\Gamma U}\right]
$$

one can define another energy scale $T^{*}$, the so-called second-stage Kondo temperature, at which the total conductance drops to zero [46]

$$
T^{*} \approx \alpha T_{K} \exp \left(-\beta T_{K} / J_{\mathrm{eff}}\right),
$$

with $J_{\text {eff }}=4 t^{2} / U$ and $\alpha, \beta$ dimensionless constants of the order of unity. This drop is the result of spin screening of the second quantum dot $[46,65]$. As can be seen in the figures, for $t=0.02 U, T^{*} \approx 10^{-6} U$, whereas for $t=0.1 U$, there is only a small resonance due to the Kondo effect at the first quantum dot, which becomes quickly suppressed as $\mathrm{T}$ is lowered. One can then estimate $T^{*} \approx T_{K}$. Consequently, $T^{*}$ is a few orders of magnitude larger in the case of $t=0.1 U$ compared to the case of $t=0.02 U$. When the coupling to the Majorana wire is turned on, it starts to play a main role in the electronic transport through the system. In the low-temperature regime one observes competitive interplay between the Kondo and Majorana physics, causing the reduction of the second dot screening, lifting conductance to the value of $e^{2} / 2 h$, which comes mainly from the spin-down contribution. Higher values of $V_{M}$ affect also the spin-up electrons, rising the conductance over $e^{2} / 2 h$ to reach its maximum value for weak hopping between the dots, see Fig. 3(a). Consequently, the existence of Majorana mode in the system has a great impact on the spin-resolved
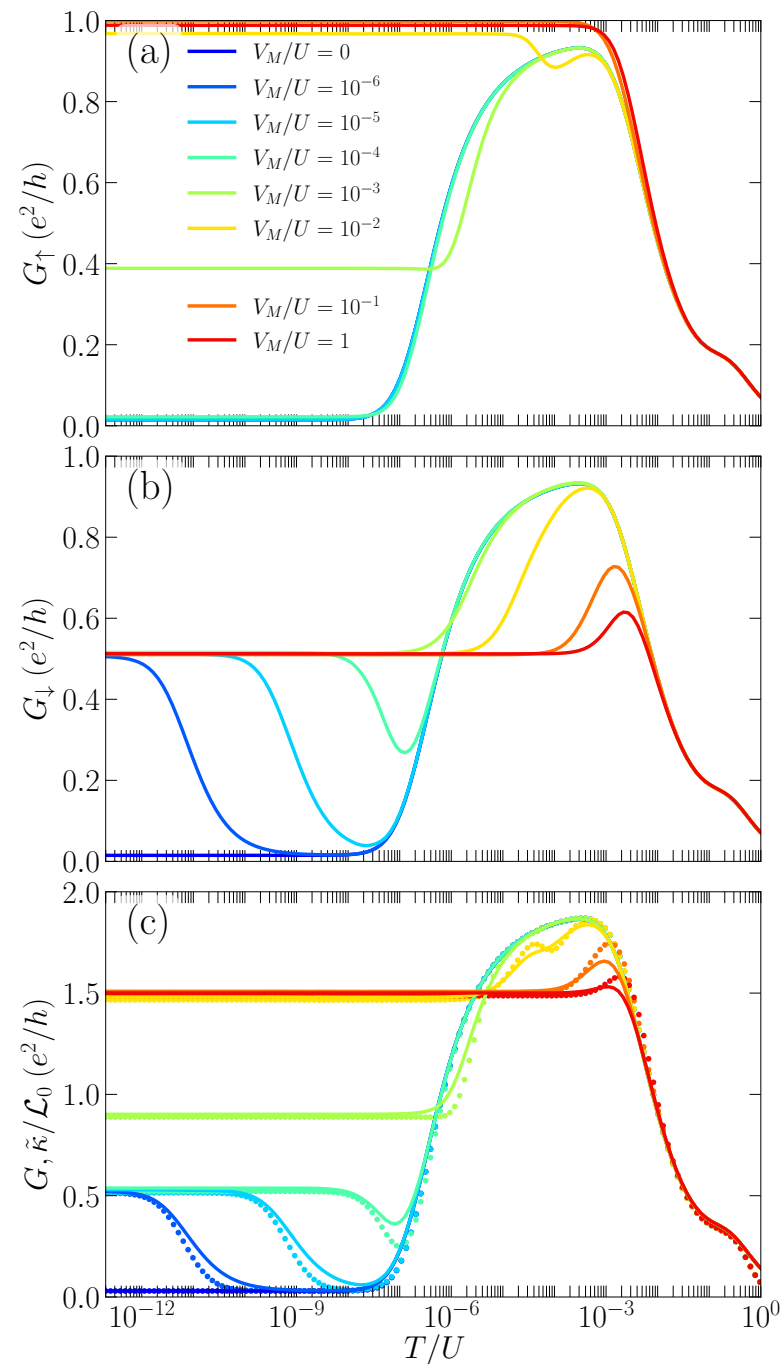

FIG. 3. (a) The spin-up $G_{\uparrow}$, (b) spin-down $G_{\downarrow}$ and (c) total conductance $G$ plotted as a function of temperature. The points in (c) present the rescaled heat conductance $\tilde{\kappa} \equiv$ $\kappa(\alpha T) /(\alpha T)$ with $\alpha=2$. The parameters are: $U=0.2$, $\Gamma=0.1 U, t=0.02 U$ and $\varepsilon_{1}=\varepsilon_{2}=-U / 3$. Please note that the curves for $G_{\uparrow}$ overlap when $V_{M} / U \lesssim 10^{-4}$, since the energy scale associated with the coupling to Majorana wire is smaller than the Kondo energy scale. This is contrary to $G_{\downarrow}$, where due to direct coupling to Majorana wire quantum interference results in suppression of the second stage of the Kondo screening, lifting the conductance, already for small coupling $V_{M} / U \approx 10^{-6}$.

transport channels, utterly destroying the second stage of the Kondo effect, which can be seen as the rise of the total conductance from $e^{2} / 2 h$ to $3 e^{2} / 2 h$ in the low temperature regime, which happens for $V_{M} / U \gtrsim 10^{-3}$, see Fig. 3(c). The maximum of the conductance develops for $T^{*} \lesssim T \lesssim T_{K}$, and is being reduced due to spin-down electrons contribution, which are more affected within the whole energy spectrum.

Enhancing the inter-dot interaction (see Fig. 4), one can observe that the competition between the coupling to 

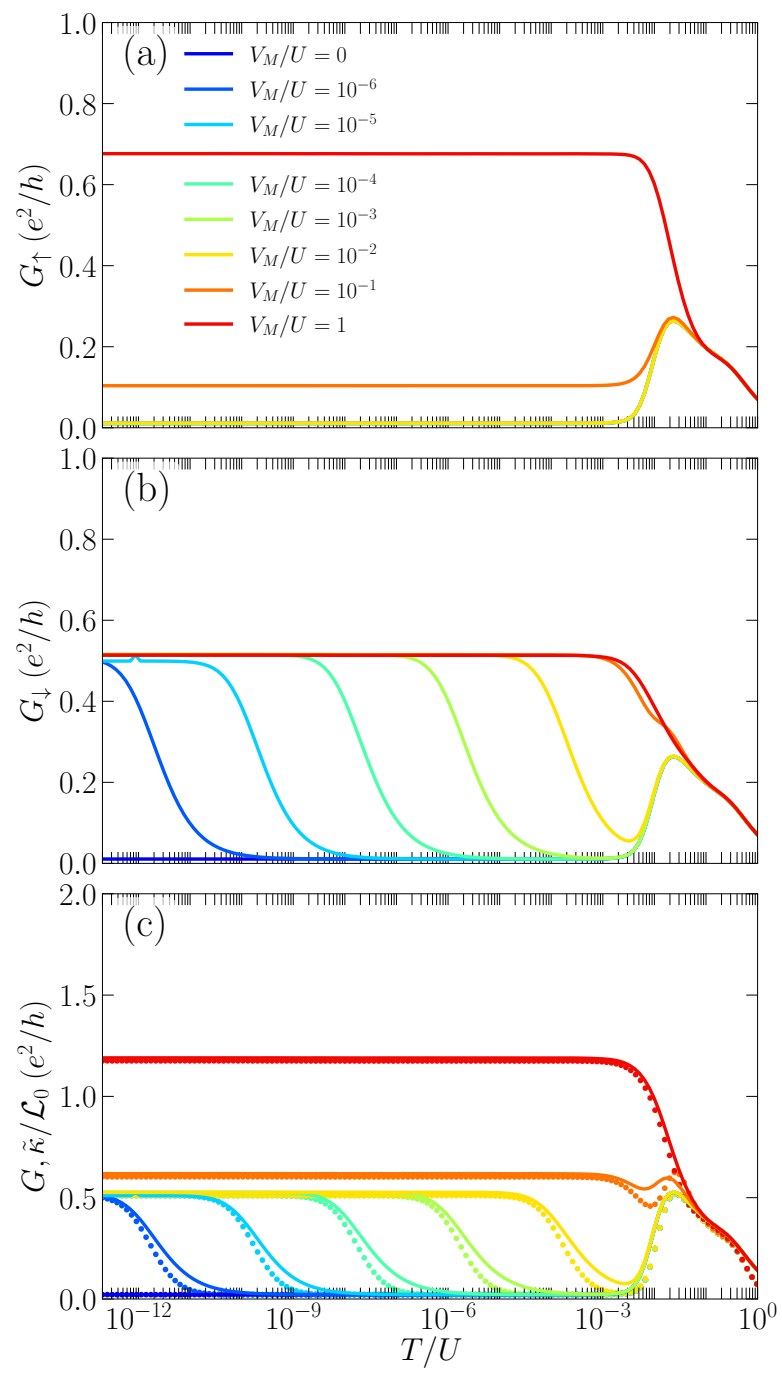

FIG. 4. The same as in Fig. 3 calculated for $t=0.1 U$.

the Majorana wire and the hopping becomes more fierce, which is especially visible in the spin-down component of $G$ and the total conductance of the system. In this regime $G_{\downarrow}$ and, thus, $G$ are being raised to $e^{2} / 2 h$ at the characteristic energy scale $\omega \sim \Gamma_{M}$ [44]. For larger values of $V_{M}$, contrary to the case of $t=0.02 U$, the Kondo effect does not develop fully even when $V_{M}$ is on the order of $U$, which is due to the formation of a molecular singlet state between the dots with binding energy of the order of the Kondo temperature, see Fig. 4.

Let us now focus on Figs. 3(c) and 4(c) where the rescaled thermal conductance in the units of temperature, $\tilde{\kappa} \equiv \kappa(\alpha T) /(\alpha T)$, is plotted (dotted lines) with colors corresponding to the values of $V_{M} / U$ of the charge conductance. Comparing the rescaled thermal conductance and the charge conductance allows us to analyze the Wiedemann-Franz law [86], which states that $\kappa / T=G \mathcal{L}_{0}$, with $\mathcal{L}_{0}=\pi^{2} / 3$.

It has been shown that in transport through the Majorana wire in different configurations the Wiedemann-
Franz law is violated [57]. Also, in a more general case of a multi-terminal superconducting island, the violation of the Wiedemann-Franz law has been proposed as a means to detect topological character of the superconductor [58]. On the contrary, it has been demonstrated that for single [52] and double quantum dot setup without Majoranas [65], a modified Wiedemann-Franz law can be introduced, $\mathcal{L}_{0}^{-1} \kappa(\alpha T) /(\alpha T)=G$, which is satisfied with good accuracy up to temperatures $T \sim U$. The key result we show here is that such modified law, quite surprisingly, also persists in the presence of coupling to topological superconducting wire. As can be seen in Figs. 3(c) and 4(c), with $\alpha \approx 2$, the rescaled thermal conductance $\tilde{\kappa}$ retraces the charge conductance even for higher values of $V_{M}$. In particular, the rescaled heat conductance in the secondstage Kondo regime rises to the quarter of its maximal value. This proves that whatever fractionalization of the effective particles takes place in the DQD structure, it is the same for charge and heat carriers, i.e. no separation of charge and heat transport takes place. This is in contrast to results obtained in Refs. [58] and [87], where excitations of the superconducting island in the topologically non-trivial phase are composed partially of electrons and partially of Andreev-reflected holes, such that the heat and charge currents are no longer simply proportional. This apparent difference is a consequence of the heat and charge current not flowing through the topological superconductor in the setup proposed here. Instead, heat and charge carriers in DQD structure have a Fermi-liquid character, and behave qualitatively similar to the non-interacting case discussed in Sec. III. The coupling to the Majorana mode leads to interferometeric effects, in particular, universal fractional values of both conductances, yet the local spectrum structure of DQD remains qualitatively different than inside the topological superconductor, in particular, the mutual coupling of heat and charge transfers is not altered.

With the Wiedeman-Franz law qualitatively correct in the proposed setup, in the following sections we proceed to pinpoint the signatures of the Majorana modes presence in other spin-resolved transport characteristics of the device, such as the current spin polarization and the (spin) Seebeck effect.

\section{B. Majorana-induced current polarization}

Maintaining our focus on the conductance, before the behavior of the Seebeck coefficient is discussed, let us take a gander on Fig. 5, where the current spin polarization is plotted as a function of $V_{M}$ for multiple values of the DQD level positions for weak and strong hopping between quantum dots in the low-temperature regime with $T=10^{-10} U$. The spin polarization of the current can be defined as

$$
\mathcal{P}=\frac{G_{\uparrow}-G_{\downarrow}}{G_{\uparrow}+G_{\downarrow}} .
$$



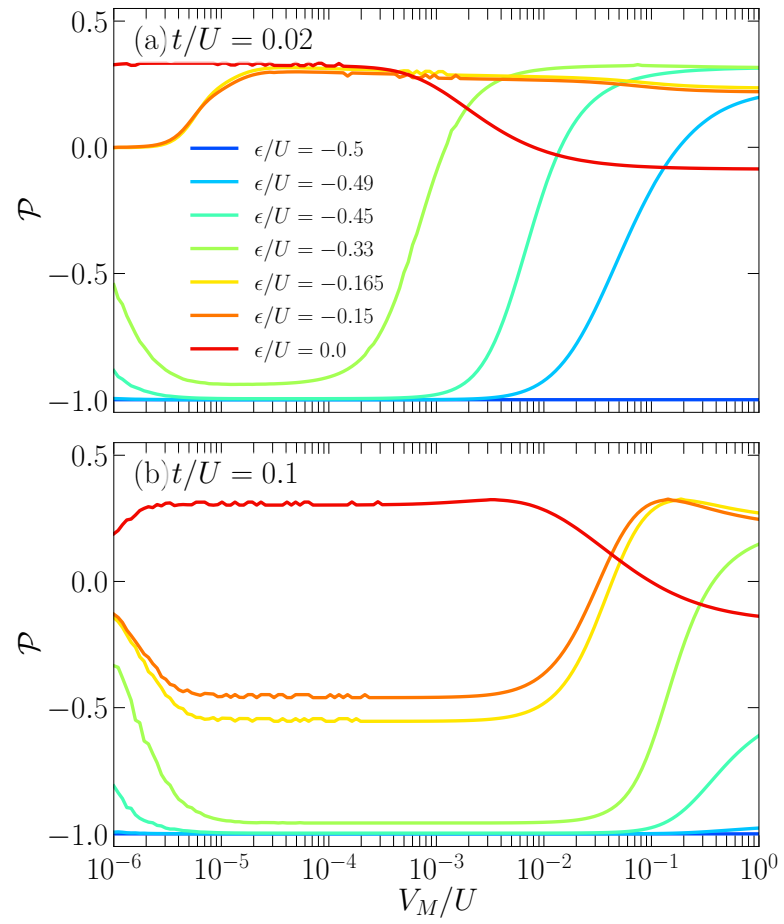

FIG. 5. The current spin polarization $\mathcal{P}$ as a function of $V_{M}$ (note the logarithmic scale) for multiple values of DQD level positions $\varepsilon_{1}=\varepsilon_{2} \equiv \varepsilon$ out of the particle-hole symmetry point, calculated for (a) $t=0.02 U$ and (b) $t=0.1 U$. The other parameters are the same as in Fig. 3 with $T=10^{-10} U$. Note that due to very low values of integrals $L_{n \sigma}$ there are oscillations in $\mathcal{P}$ for $V_{M} \lesssim 10^{-3} U$ that should be considered as numerical error, which does not affect qualitative behavior of presented results.

Usually, in systems such as quantum dots, finite spin polarization of the current is accompanied with suppression of the Kondo effect [88]. External magnetic field [89] or ferromagnetic leads [90], which usually introduce the spin imbalance of current carriers, split the Kondo peak and eventually cause it to disappear. In the case of the system studied here, the spin polarization is associated with the coupling to topological superconductor and it is not necessarily accompanied with suppression of the Kondo state.

In Fig. 5(a) the current spin polarization for weak hopping $t / U=0.02$ is shown. First, we note that $\mathcal{P}=-1$ when the system is at the particle-hole symmetry point, $\varepsilon=-U / 2$, within the whole range of $V_{M}$. This is due to the fact that $G_{\uparrow}$ is then suppressed due to the two-stage Kondo effect, while $G_{\downarrow}=e^{2} / 2 h$, due to the quantum interference with the Majorana wire. Let us now analyze what happens when detuning from $\varepsilon=-U / 2$ is turned on. Starting with the case of $\varepsilon=-0.49 U$, for low values of $V_{M}, G_{\uparrow}$ is suppressed due to the second-stage Kondo effect (note that $T=10^{-10} U$ ), but finite value of $V_{M}$ affects the Kondo state giving rise to small but finite $G_{\downarrow}$. It results in a perfect negative spin polarization $\mathcal{P} \approx-1$, quite a remarkable feature in the fully screened Kondo regime, stressing its unusual character. This result holds up to $V_{M} \approx 10^{-2} U$, when further increase of $V_{M}$ eventually affects the spin-up conductance restoring the firststage Kondo effect when $V_{M} \gtrsim 10^{-2} U$. This leads to an increase of spin polarization to a moderate positive value for $V_{M} \approx U$.

Shifting the DQD levels further from the particlehole symmetry point, the effect of perfect spin polarization with $\mathcal{P} \approx-1$ becomes distorted, which is due to Majorana-induced splitting, which grows with detuning the levels $[39,41]$, as well as the fact that the lowtemperature second-stage Kondo conductance attains a finite value. As a consequence, the spin-up conductance is being lifted, while the spin-down channel remains approximately intact. Moreover, smaller values of $V_{M}$ are now needed to increase $G_{\uparrow}$. This manifests itself as a shift in sign change of $\mathcal{P}$ toward lower values of the Majorana interaction. Such behavior is maintained until $\varepsilon$ crosses the point where $T^{*} \approx \Gamma_{M}$, which appears at $\varepsilon \approx-0.25 \mathrm{U}$. For $\varepsilon$ above this value, the spin polarization becomes suppressed, $\mathcal{P} \approx 0$, for low coupling to the superconducting nanowire. Increasing $V_{M}$ leads to the spin polarization sign change, setting it positive at $\mathcal{P} \approx 0.25$. This abrupt change is due to entering the regime, at which both spin-up and spin-down channels take part in transport, with $G_{\downarrow}=e^{2} / 2 h$ for a significant range of $V_{M}$. Finally, reaching the resonance point $\varepsilon \approx 0$, low Majorana coupling limit exhibits a rather strong positive spin polarization, while for larger values of $V_{M}, \mathcal{P}$ becomes almost suppressed due to comparable contribution of both spin channels.

Considering the case of strong hopping between quantum dots shown in Fig. 5(b), one can see that the spin polarization takes values similar to the ones already discussed, however, the range of $V_{M}$ where e.g. $\mathcal{P}<0$ develops is now extended. Strong enough $t$ blocks the conductance in both spin channels, nevertheless, the interplay of Kondo and Majorana physics can result in an increase of conductance and the corresponding behavior of $\mathcal{P}$. While $G_{\uparrow}$ is damped for almost whole energy level spectrum, $G_{\downarrow}$ behaves similarly as in the Kondo regime, being half-suppressed for $V_{M} \gtrsim 10^{-5} U$. It leads to almost full negative spin polarization for dot level position between $\varepsilon \approx-0.66 U$ and $\varepsilon \approx-0.33 U( \pm U / 6$ around the particle-symmetry point). Above (below) the aforementioned range, $G_{\uparrow}$ starts to increase, lifting $\mathcal{P}$ towards zero, finally changing the sign of spin polarization at resonance.

To sum this section up, we have shown that the finite coupling to the Majorana wire results in nonzero spin polarization. Furthermore, this spin polarization can have large negative values and it changes sign depending on the strength of coupling to topological superconductor and the position of the double dot levels.

\section{Majorana-induced thermopower sign change}


The following section will expand the discussion to another important thermoelectric effect - the thermopower (or the Seebeck coefficient). In general, for a Fermi liquid (FL) at sufficiently low temperature $T, S$ follows the Mott formula based on the Sommerfeld expansion, with its characteristic $S \sim T$ dependence [77], cf. Eq. (14). This is valid when $T$ is smaller than all energy scales characterizing given FL. Typically, Fermi liquid features a peak in $\mathcal{T}(\omega)$ at $\omega=\omega_{0}$ corresponding to quasi-particle energy level, of width $\Gamma_{\text {eff }}$ inversely proportional to the quasi-particle life time. For $T \ll \omega_{0}, \Gamma_{\text {eff }}$, the linear dependence of thermopower follows from the Mott formula (14). On the other hand, for $T \gg \omega_{0}, \Gamma_{\text {eff }}$, the integrals in Eq. (5) in fact average all the $\omega$-dependence out and the $T^{-1}$ term from Eq. (7) remains [91]. This leaves $S(T)$ no choice, but to exhibit a peak at $T \sim \omega_{0}, \Gamma_{\text {eff }}$, with a sign depending on which slope of $\mathcal{T}(\omega)$ is present at $\omega=0$.

This picture repeats itself in a series of peaks of alternating sings in T-shaped double quantum dots, where the two-stage Kondo effect is present [65]. Three FLs are involved there: at elevated $T \sim U$, even QD levels deep below Fermi level contribute to the transport. Transport is suppressed in the Coulomb blockade regime $T_{K} \ll T \ll U$, where relevant excitations do not form a FL, only to rise again at $T$ below $T_{K}$, when FL character of relevant degrees of freedom is restored and another peak appears in $S(T)$. Similar scenario happens for $T<T^{*}$, then only the spectral function of the second dot exhibits a peak, while $\mathcal{T}(\omega)$ shows the corresponding dip, leading to a peak in $S(T)$ of opposite sign.

\section{Temperature dependence}

The situation becomes even more interesting in the presence of topological superconductor. In Fig. 6 the spin-resolved Seebeck coefficient for multiple values of $V_{M}$ and two selected values of $t$ is presented. For relatively weak hopping $t=0.02 U$ (see the left column of Fig. 6), coupling the system with Majorana mode causes the low-temperature sign change of the spin-down and total thermopower, see Figs. 6(c) and (e). This picture directly reflects the behavior of the conductance presented in Fig. 3, where an increase of $G$ with lowering $T$ is observed. For $10^{-6} U \lesssim V_{M} \lesssim 10^{-4} U$, i.e. when $\Gamma_{M}$ is smaller than Kondo-related energy scales, there is the corresponding minimum in $S_{\downarrow}$ and (thus in) $S$ occurring at $T \sim \Gamma_{M}$. At this temperature, the resurgence of conductance $G$ is observed, and according to the Mott formula, a sudden change of the thermopower can be expected. Stronger coupling with the superconducting nanowire results in shallowing of the minimum, which one can relate to stronger Majorana-Kondo competition in the system. For values of $V_{M}$ up to about $10^{-4} U$, the low-temperature transport is dependent only on the spin-down electron channel. However, increasing the interaction between the Majorana mode and the second quantum dot, additional contribution from spin-up

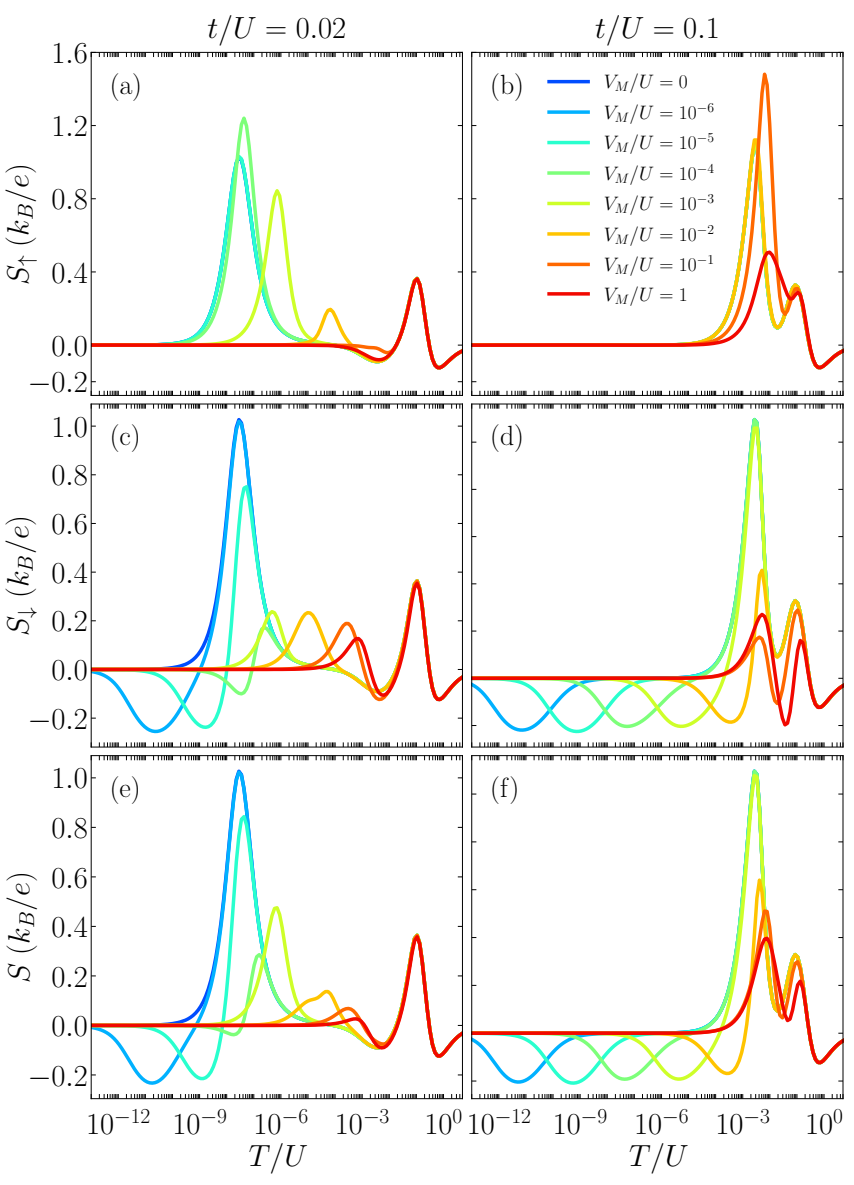

FIG. 6. The Seebeck coefficient $S$ as a function of temperature for (left column) $t=0.02 U$, (right column) $t=0.1 U$ and multiple values of $V_{M}$ as indicated in the legend. The other parameters are the same as in Fig. 3.

electrons emerges, see Fig. 6(a). The minimum of thermopower is then being reduced and only positive peaks of the Seebeck coefficient, combined from the spin-up and spin-down electron channels, remain. Moreover, stronger coupling to the Majorana wire increases the second-stage Kondo temperature $T^{*}$, which results in shifting of thermopower peak toward higher temperatures. We also note that the behavior of the Seebeck coefficient at higher temperatures, i.e. $T \gtrsim T_{K}$, is consistent with that predicted for single quantum dots [52, 92].

The quantum interference with Majorana zero-energy mode is much better visible in the case of strong hopping between the dots, which is presented in the right column of Fig. 6. In this regime one can notice a series of minima of the Seebeck coefficient corresponding to $T \sim \Gamma_{M}$. As long as $V_{M}$ is not strong enough to dominate the transport in the system, the prevailing spin-down electron contribution is observed, where the coupling to the superconducting nanowire results in increased conductance to the total value of $G=e^{2} / 2 h$. Such destructive behavior relative to the second-stage Kondo effect plays a main role in the sign changes of the thermopower. Similar to the pre- 


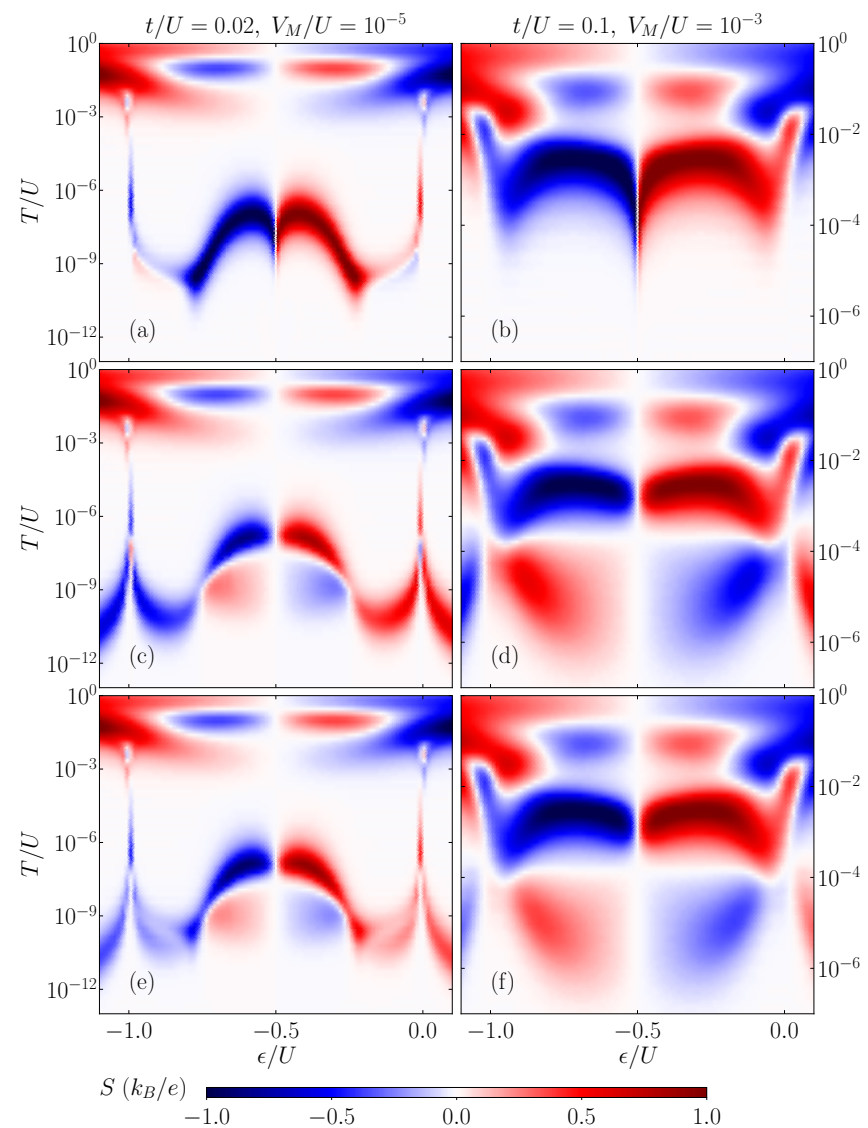

FIG. 7. The (a,b) spin-up, (c,d) spin-down, and (e,f) total Seebeck coefficient for $t=0.02 U$ and $V_{M} / U=10^{-5}$ (left column) and $t=0.1 U$ and $V_{M} / U=10^{-3}$ (right column) as a function of temperature $T$ and position of the quantum dot levels $\varepsilon_{1}=\varepsilon_{2}=\varepsilon$. Note different temperature scale for each column. The other parameters are the same as in Fig. 3.

vious case, when the coupling to the Majorana wire is of the order of or above the Kondo energy scales, it also affects the spin-up electrons, resulting in a large maximum, which is visible both in $S_{\uparrow}$ and $S_{\downarrow}$. Consequently, the total thermopower features a maximum around $T \sim T_{K}$, see Fig. 6(f).

\section{Gate voltage dependence}

Figure 7 presents the Seebeck coefficient as a function of temperature $T$ and double dot energy levels, with $\varepsilon_{1}=\varepsilon_{2}=\varepsilon$. Left column of Fig. 7 shows the results corresponding to the weak interdot hopping, while the right column is for the case of strong $t$. The first (second) row depicts the spin-up (spin-down) thermopower, while the bottom row presents the total Seebeck coefficient. First of all, one can note that thermopower changes sign when crossing the particle-hole symmetry point, which is a natural consequence of the fact that the type of majority charge carriers changes from electrons to holes as $\varepsilon$ crosses $-U / 2$. Moreover, in the behavior of thermopower one can generally distinguish four regimes determined by the relevant energy scales: $\Gamma, T_{K}, T^{*}$ and $\Gamma_{M}$. Within $-0.75 U \lesssim \varepsilon \lesssim-0.25 U$, they outline an interesting behavior in total thermopower of the system. Note that because $T^{*}$ strongly depends on $t$, the corresponding low temperature behavior in the case of weak hopping is much more extended in the case when the hopping is strong, cf. the left and right column of Fig. 7. Let us start the discussion with the former case. One can see that in the Coulomb blockade regime there is a characteristic peak at $T \approx \Gamma$, which does not depend on $V_{M}$ [cf. Fig. 6], but changes sign near the resonance. Then, with lowering the temperature, the first stage of the Kondo effect occurs, where the first Fermi liquid forms and the charge transport through the first quantum dot is relatively strong. For this energy range, the thermopower exhibits a small sign change with the minimum around $T \approx T_{K}$. Shifting to the lower temperature regime, where $T^{*} \lesssim T \lesssim T_{K}$, the total thermopower gets suppressed, while the total conductance reaches a maximum signaling the full development of the first-stage Kondo regime.

An interesting behavior is revealed when the temperature drops below $T^{*}$. In that case one can distinguish the regime limited by $T^{*}$ and $\Gamma_{M}$ (for assumed parameters $\Gamma_{M} \approx 10^{-9} U$ ), where a sign change, not visible for $V_{M}=0$ [cf. Fig. 6(e)], is observed. In this parameter space one can observe consecutive thermopower minima and maxima. It can be related to charge conductance which is affected by the coupling to the Majorana mode, and considering the low-temperature limit, one can understand that with the Mott formula. Therefore, these peaks are related to $\Gamma_{M}$ and $T^{*}$, at which the change in transmission coefficient $\mathcal{T}(\omega)$ is observed, and the sign change of total thermopower stems from the derivative of $\mathcal{T}(\omega)$. When the temperature drops below $\Gamma_{M}$, one can see the result of Majorana-induced half-destruction of the second-stage of the Kondo effect, hence the thermopower becomes suppressed.

When the hopping between the dots is stronger [see Figs. $7(\mathrm{~b}, \mathrm{~d}, \mathrm{f})$ for $S_{\uparrow}, S_{\downarrow}$ and $S$, respectively], the Kondo effect hardly develops when the system is not coupled with the Majorana zero mode. However, strong enough coupling to the superconducting nanowire induces additional minimum in thermopower for $\varepsilon<-U / 2$ (maximum for $\varepsilon>-U / 2$ ), which forms at $T \approx \Gamma_{M}$. The energy at which a sign change occurs with further lowering the temperature is equal in almost the entire range of DQD level position, see Fig. 7(f). The parameter space at which the sign change is observed is related to the minimum of conductance and Majorana-induced increase of $G_{\downarrow}$ at low temperatures, cf. Fig. 4.

We also note that the colormaps shown in Fig. 7 allow one to easily identify the new behavior associated with the presence of Majorana modes, which is mostly revealed in the low-temperature behavior of $S_{\downarrow}$ and then, consequently, in the corresponding dependence of $S$.

The sign change of the thermopower is undoubtedly 
a notable result of this paper, showing unconventional effect arising from the coupling of the double quantum dot to topological superconductor.

\section{Spin Seebeck effect due to Majorana proximity}

Since it is assumed that the Majorana mode couples to only one of the spin components in the second quantum dot, it breaks the spin symmetry of the system and may thus give rise to interesting spin-resolved thermoelectric phenomena, such as nonzero spin thermopower (or spin Seebeck effect). If the electrodes are characterized by long spin relaxation time, spin accumulation may build up in the contacts, such that the voltage generated by the temperature gradient could become spin dependent $\Delta V_{\sigma}$. In this case, the linear response current in the spin channel $\sigma$ is given by [93]

$$
I_{\sigma}=e^{2} L_{0 \sigma} \Delta V+\eta e^{2} L_{0 \sigma} \Delta V_{S}-\frac{e}{T} L_{1 \sigma} \Delta T
$$

where $\Delta V_{S}=\left(\Delta V_{\uparrow}-\Delta V_{\downarrow}\right) / 2$ and $\eta=+1$ for $\sigma=\uparrow$ and $\eta=-1$ for $\sigma=\downarrow$. There exist two definitions of the spin thermopower in the literature [93], depending on the experimental implementation. Defining the spin thermopower $S_{S}$ assuming open circuit conditions (vanishing of both charge and spin currents), one gets [92, 93] $S_{S}=\left(S_{\uparrow}-S_{\downarrow}\right) / 2$, while for $S$ one finds $S=\left(S_{\uparrow}+S_{\downarrow}\right) / 2$. On the other hand, if one requires that only the spin current vanishes on the condition that the voltage gradient is zero $\Delta V=0$, the spin thermopower is given by [94]

$$
S_{S}=-\frac{1}{e T} \frac{L_{1 \uparrow}-L_{1 \downarrow}}{L_{0}}
$$

In the following we study the behavior of the spin thermopower defined in the latter formula. We also note that since we have already discussed in detail the spinresolved components of thermopower $S_{\sigma}$ in previous sections, the behavior of thermopower determined assuming open circuit conditions, which is given either by a sum or difference of these components, can be anticipated from previous results.

\section{Temperature dependence}

Figure 8 presents the spin thermopower $S_{S}$ plotted as a function of temperature $T$ for multiple values of $V_{M}$, for both weak and strong value of hopping $t$ between the quantum dots. Consider first the case where $t=0.02 U$ [see Fig. 8(a)]. Starting with $V_{M}=0$, the spin Seebeck effect does not develop. Increasing the coupling to Majorana zero mode, a single positive peak appears at $T \approx \Gamma_{M}$, and remains when increasing $V_{M}$ until $\Gamma_{M} \approx T^{*}$. Moreover, while the height of this maximum is qualitatively similar for different values of $V_{M}$, its width becomes sharper (on logarithmic scale) as $\Gamma_{M}$ becomes of
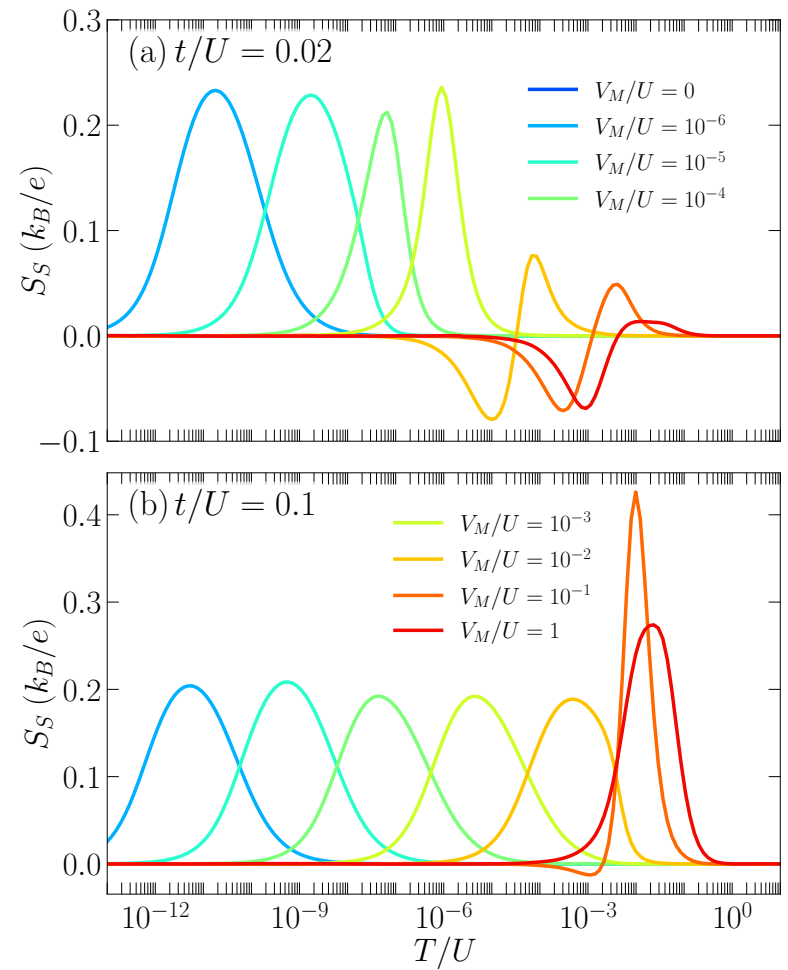

FIG. 8. The spin Seebeck coefficient $S_{S}$ plotted as a function of $T$ for different values of $V_{M}$ as indicated and for (a) $t=$ $0.02 U$ and (b) $t=0.1 U$. The other parameters are the same as in Fig. 3.

the order of $T^{*}$. We can associate this phenomenon with the interplay of the Majorana physics and the two-stage Kondo effect, where $\Gamma_{M}$ and $T^{*}$ energy scales are relevant. They limit the conductance gap due to the second quantum dot screening, what quantitatively affects the width of the peak. Increasing $V_{M}$, the gap becomes narrower as the difference between $T^{*}$ and $\Gamma_{M}$ decreases. As a result, one observes the maximum of spin thermopower shifting toward higher temperatures. When $V_{M}$ is so large that $\Gamma_{M}$ reaches and exceeds $T^{*}$, see the curves for $V_{M} \gtrsim 10^{-2} U$ in Fig. 8, one observes a sudden sign change in $S_{S}$. In this regime, the spin-up contribution becomes stronger, which is also visible in the spin polarization of the current, which changes sign to positive values as $V_{M}$ grows, cf. Fig. 5(a). The height of minima (maxima) in $S_{S}$ strongly depends on the spin-down carriers of which the Majorana-Kondo interplay is visible with its characteristic half-suppression of $G_{\downarrow}$.

The case of strong interdot hopping, where $t=0.1 U$, is shown in Fig. 8(b). Now, the situation is rather different. In this case, when the double quantum dot is decoupled from the topological nanowire, the Kondo effect hardly develops due to strong singlet state, which forms between the dots. Nonetheless, as in the situation of weak hopping discussed above, turning on the coupling to Majorana zero mode changes this behavior significantly. Finite $V_{M}$ gives rise to quantum interfer- 


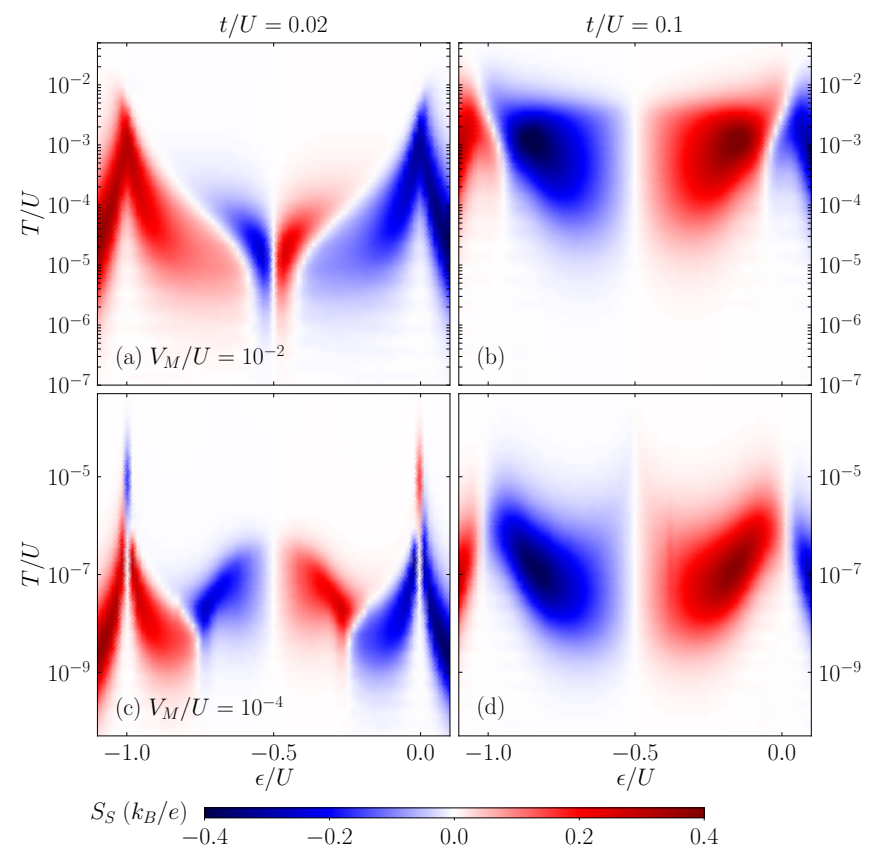

FIG. 9. The spin Seebeck effect as a function of $T$ and $\varepsilon$ for $(\mathrm{a}, \mathrm{c}) t=0.02 U$ and $(\mathrm{b}, \mathrm{d}) t=0.1 U$. The first row $(\mathrm{a}, \mathrm{b})$ is calculated for $V_{M}=10^{-2} U$, while the second row (c,d) presents $S_{S}$ for $V_{M}=10^{-4} U$. The other parameters are the same as in Fig. 3.

ence in the spin-down channel, resulting in $G_{\downarrow}=e^{2} / 2 h$. This leads to a peak visible in spin thermopower. The position of this peak follows the increase of $V_{M}$, with approximately equal height and width (on the logarithmic scale) until $\Gamma_{M} \approx 10^{-2} U$, where both the width and height become changed, see Fig. 8(b).

\section{Gate voltage dependence}

Figure 9 presents the behavior of the spin Seebeck effect as a function of the double dot levels $\varepsilon=\varepsilon_{1}=\varepsilon_{2}$ and temperature $T$. The left (right) column is calculated in the case of weak (strong) hopping between the dots, while the first (second) row presents the data for $V_{M}=10^{-2} U\left(V_{M}=10^{-4} U\right)$. Let us start the discussion with the case of weak interdot hopping. When $V_{M}=10^{-2} U$, the spin-resolved conductance [cf. Fig. 3] exhibits changes for $T \gtrsim 10^{-5} U$, while at lower temperatures it takes constant value, with $G_{\downarrow} \approx e^{2} / 2 h$ and $G_{\uparrow} \approx e^{2} / h$. Revoking the Mott formula, this helps to understand the temperature range where non-zero spin Seebeck effect can emerge. As one can see in the figure, around the particle-hole symmetry point $S_{S}$ is either positive or negative depending on the sign of detuning from $\varepsilon=-U / 2$. In the case of $\varepsilon>-U / 2, S_{S}$ exhibits a maximum which moves to higher temperatures as the detuning grows. Moreover, for $\varepsilon \gtrsim-0.4 U$, a sign change in the $T$-dependence of the spin Seebeck effect develops, cf. also Fig. 8(a). When the detuning from the particle-hole symmetry point grows, the contributions from the spinup and spin-down channels become comparable, which results in suppression and the corresponding reversal of the spin thermopower. Such a reversal is visible in a narrow range of DQD level position, $-0.4 U \lesssim \varepsilon \lesssim-0.2 U$ and $-0.8 U \lesssim \varepsilon \lesssim-0.6 U$, see Fig. 9(a). Otherwise $S_{S}$ as a function of $T$ exhibits a large negative (positive) peak for $\varepsilon \gtrsim-0.2 U(\varepsilon \lesssim-0.8 U)$, whose position strongly depends on $\varepsilon$. When the coupling to Majorana wire is weaker, see the case of $V_{M}=10^{-4} U$ presented in Fig. 9(c), the behavior of $S_{S}$ becomes greatly modified. First of all, the characteristic temperature range where spin thermopower emerges is shifted to lower $T$, which is due to the fact that the characteristic energy scale associated with the presence of Majorana mode is now reduced. Moreover, the behavior around the particle-hole symmetry point is extended over a wider range of $\varepsilon$. This results from the fact that the Majorana energy scale is smaller and the relevant interplay between Majorana-induced interference and the Kondo correlations now takes place in larger parameter space of the system.

The situation when the interdot hopping is strong is shown in the right column of Fig. 9. First of all, one can see that the relevant behavior of $S_{S}$ is shifted to larger temperatures, since now the second-stage Kondo temperature is of the order of the Kondo temperature. Furthermore, contrary to the weak hopping limit, there is no effect where $S_{S}$ changes sign within the Coulomb valley. Instead, one can observe a single positive (or negative for $\varepsilon<-U / 2$ ) peak of width depending on $\varepsilon$, whose extremum shifts toward higher temperatures with detuning from the particle-hole symmetry point. Such behavior results from the corresponding dependence of the spin-resolved conductance [cf. Fig. 4], which changes on the scale of $T \sim T^{*}$ and reaches a constant value for lower temperatures. This peak for chosen parameter space reaches its maximum (minimum) for $\varepsilon \approx-0.15 \mathrm{U}$ $(\varepsilon \approx-0.85 U)$. Shifting away from this point toward the symmetry point, $S_{S}$ diminishes and the peak widens. A similar behavior can be observed when the Majorana coupling is smaller, see the case of $V_{M}=10^{-4} U$ in Fig. 9(d), but now the temperature range where the spin thermopower can be observed is shifted to lower temperatures. Otherwise, the qualitative behavior is similar.

To conclude, the generation of the spin thermopower is another important result of this paper. Together with the spin polarization, these effects provide insight into the spin-dependent properties, characteristic of the model presented in this paper and stemming from the coupling to topological superconductor.

\section{SUMMARY}

We have studied the spin-resolved electrical and thermal signatures of the interplay between the Majorana and Kondo physics in the case of T-shaped double quan- 

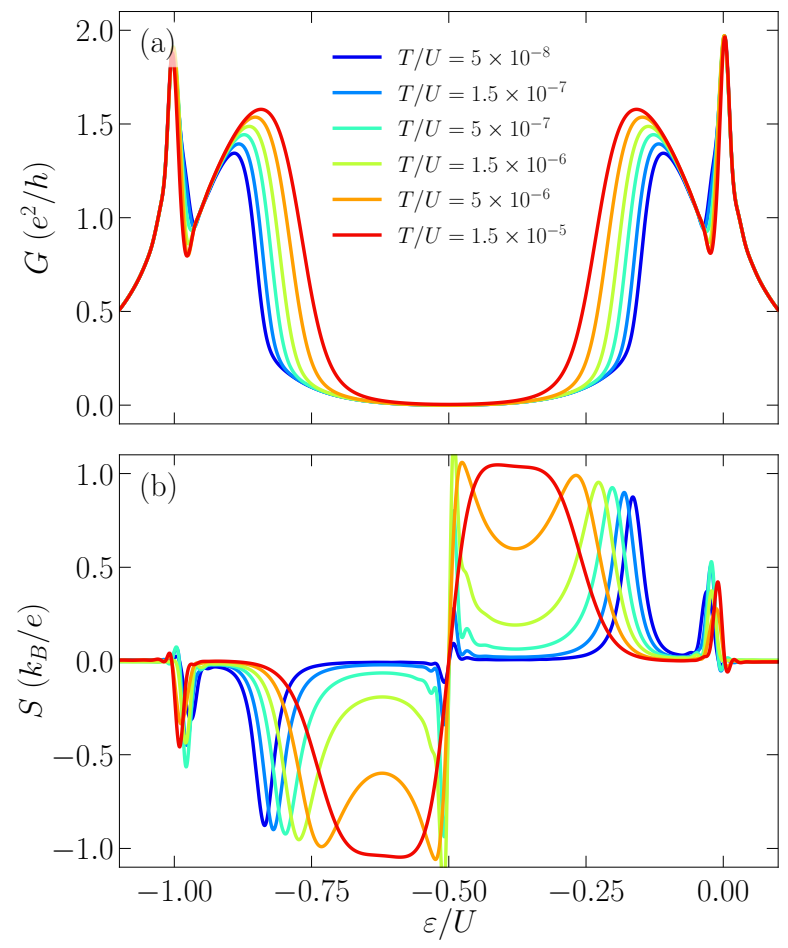

FIG. 10. The conductance and thermopower as a function of $\varepsilon=\varepsilon_{1}=\varepsilon_{2}$ for different values of $T$ and hopping between the dots $t / U=0.033$ in the case when the system is not coupled to the superconducting nanowire, $V_{M}=0$.

tum dots attached to normal contacts and to topological superconductor hosting Majorana zero energy modes. To accurately resolve the transport behavior in the full parameter space we have used the density-matrix numerical renormalization group method. We have determined the behavior of the conductance, current spin polarization, heat conductance as well as the Seebeck and spin Seebeck coefficients, focusing on the parameter space where the system exhibits the Kondo correlations. In our analysis, we have considered two specific cases of interdot hopping: the weak hopping regime, where the two-stage Kondo effect develops, and the strong hopping regime, where a molecular singlet state forms between the dots allowing for only an onset of Kondo state in the system.

Analyzing the temperature dependence of the linear conductance, we have shown that quantum interference with Majorana wire breaks the second-stage of Kondo screening raising the conductance to a fractional value of $G=e^{2} / 2 h$. We have also shown that the presence of Majorana mode gives rise to finite spin polarization of the current, which strongly depends on the magnitude of coupling to Majorana wire and the position of the double dot energy levels.

As far as thermometric properties are concerned, we have demonstrated that the system exhibits a modified Wiedemann-Franz law, which is satisfied by the heat conductance at a rescaled temperature, also in the presence of coupling to topological superconductor. Furthermore, we
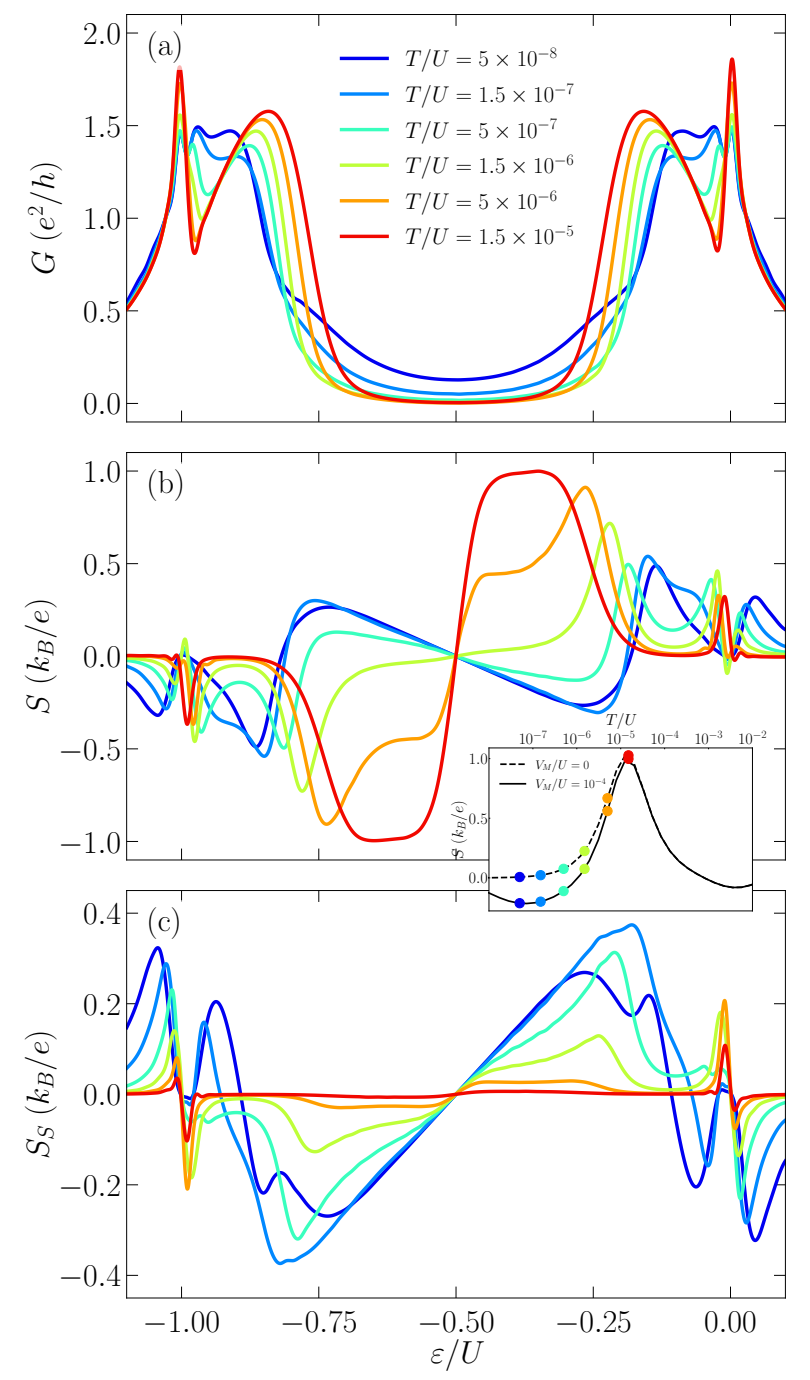

FIG. 11. The conductance, thermopower and spin thermopower with the same parameters as in Fig. 10, but with $V_{M} / U=10^{-4}$. The inset shows the thermopower as a function of temperature, calculated for $V_{M} / U=0$ and $V_{M} / U=$ $10^{-4}$. The dots' colors correspond to the temperatures shown in the legend of both figures. As can be seen, for $T / U \gtrsim 10^{-5}$, $S$ hardly depends on $V_{M}$, and these values of $T / U$ are not shown. Panel (c) displays the spin thermopower, which is induced by the coupling to Majorana mode, thus this panel is not shown for $V_{M}=0$ in Fig. 10 .

have analyzed the temperature dependence of the spinresolved Seebeck effect and shown that the thermopower exhibits additional sign change associated with the presence of Majorana zero energy mode in the system. Finally, assuming long spin relaxation time in the contacts, we have examined the behavior of the spin Seebeck coefficient, which emerges due to the presence of coupling to topological superconductor and is a unique property of the considered Majorana-double quantum dot setup. We have demonstrated that the spin thermopower nicely reveals the features resulting from the interplay of strong electron correlations and Majorana-induced interference. 
We would like to note that although the spin-resolved results presented in this paper are challenging from experimental point of view, they have a clear fundamental relevance and are crucial to fully understand the system's behavior, especially how the coupling to Majorana wire affects the different spin components. However, as far as experimental aspects are concerned, we believe that the spin-resolved measurements could be performed by using e.g. spin-polarized STM [95] or by attaching spin filters made by e.g. ferromagnetic electrodes [96]. We hope that our work will foster further efforts in spin-resolved transport spectroscopy of hybrid nanostructures.

Finally, to emphasize the effects stemming from the presence of Majorana quasiparticles and allow for their clear identification, in Figs. 10 and 11 we present the dependence of the conductance and the (spin) Seebeck coefficient on the position of dots levels for different values of temperatures. Figure 10 presents the data in the absence of coupling to Majorana wire, while Fig. 11 shows the results in the presence of coupling to topological superconductor. Indeed, the sign change of the thermopower due to finite $V_{M}$ is now clearly visible. Moreover, nonzero spin Seebeck coefficient emerges only when the Majorana mode is present in the system.

\section{ACKNOWLEDGMENTS}

This work was supported by the National Science Centre in Poland through the Project No. 2018/29/B/ST3/00937. The computing time at the Poznań Supercomputing and Networking Center is acknowledged. KPW acknowledges support from A. von Humboldt Foundation.
[1] E. Majorana, Teoria simmetrica dell'elettrone e del positrone, Nuovo Cim. 14, 171 (1937).

[2] A. Y. Kitaev, Unpaired Majorana fermions in quantum wires, Phys. Usp. 44, 131 (2001).

[3] A. Yu. Kitaev, Fault-tolerant quantum computation by anyons, Ann. Phys. 303, 2 (2003).

[4] L. Fu and C. L. Kane, Superconducting Proximity Effect and Majorana Fermions at the Surface of a Topological Insulator, Phys. Rev. Lett. 100, 096407 (2008).

[5] C. Nayak, S. H. Simon, A. Stern, M. Freedman, and S. Das Sarma, Non-Abelian anyons and topological quantum computation, Rev. Mod. Phys. 80, 1083 (2008).

[6] C. W. J. Beenakker, Search for non-Abelian Majorana braiding statistics in superconductors, SciPost Phys. Lect. Notes , 15 (2020).

[7] M. Z. Hasan and C. L. Kane, Colloquium: Topological insulators, Rev. Mod. Phys. 82, 3045 (2010).

[8] X.-L. Qi and S.-C. Zhang, Topological insulators and superconductors, Rev. Mod. Phys. 83, 1057 (2011).

[9] J. Wang and S.-C. Zhang, Topological states of condensed matter, Nat. Mater. 16, 1062 (2017).

[10] M. Sato and Y. Ando, Topological superconductors: a review, Rep. Prog. Phys. 80, 076501 (2017).

[11] V. Mourik, K. Zuo, S. M. Frolov, S. R. Plissard, E. P. A. M. Bakkers, and L. P. Kouwenhoven, Signatures of Majorana Fermions in Hybrid SuperconductorSemiconductor Nanowire Devices, Science 336, 1003 (2012).

[12] R. M. Lutchyn, J. D. Sau, and S. Das Sarma, Majorana Fermions and a Topological Phase Transition in Semiconductor-Superconductor Heterostructures, Phys. Rev. Lett. 105, 077001 (2010).

[13] Y. Oreg, G. Refael, and F. von Oppen, Helical Liquids and Majorana Bound States in Quantum Wires, Phys. Rev. Lett. 105, 177002 (2010).

[14] R. M. Lutchyn, E. P. A. M. Bakkers, L. P. Kouwenhoven, P. Krogstrup, C. M. Marcus, and Y. Oreg, Majorana zero modes in superconductor-semiconductor heterostructures, Nat. Rev. Mater. 3, 52 (2018).

[15] E. Prada, P. San-Jose, M. W. A. de Moor, A. Geresdi, E. J. H. Lee, J. Klinovaja, D. Loss, J. Nygård, R. Aguado, and L. P. Kouwenhoven, From Andreev to Majorana bound states in hybrid superconductor-semiconductor nanowires, Nat. Rev. Phys. 2, 575 (2020).

[16] E. J. H. Lee, X. Jiang, M. Houzet, R. Aguado, C. M. Lieber, and S. De Franceschi, Spin-resolved Andreev levels and parity crossings in hybrid superconductor-semiconductor nanostructures, Nat. Nanotechnol. 9, 79 (2013).

[17] G. Kells, D. Meidan, and P. W. Brouwer, Near-zeroenergy end states in topologically trivial spin-orbit coupled superconducting nanowires with a smooth confinement, Phys. Rev. B 86, 100503(R) (2012).

[18] D. Wang, J. Wiebe, R. Zhong, G. Gu, and R. Wiesendanger, Spin-Polarized Yu-Shiba-Rusinov States in an IronBased Superconductor, Phys. Rev. Lett. 126, 076802 (2021).

[19] D. I. Pikulin, J. P. Dahlhaus, M. Wimmer, H. Schomerus, and C. W. J. Beenakker, A zero-voltage conductance peak from weak antilocalization in a Majorana nanowire, New J. Phys. 14, 125011 (2012).

[20] F. Borsoi, K. Zuo, S. Gazibegovic, R. L. M. Op het Veld, E. P. A. M. Bakkers, L. P. Kouwenhoven, and S. Heedt, Transmission phase read-out of a large quantum dot in a nanowire interferometer, Nat. Commun. 11, 1 (2020).

[21] A. M. Whiticar, A. Fornieri, E. C. T. O'Farrell, A. C. C. Drachmann, T. Wang, C. Thomas, S. Gronin, R. Kallaher, G. C. Gardner, M. J. Manfra, C. M. Marcus, and F. Nichele, Coherent transport through a Majorana island in an Aharonov-Bohm interferometer, Nat. Commun. 11, 1 (2020).

[22] J. Avila, F. Peñaranda, E. Prada, P. San-Jose, and R. Aguado, Non-hermitian topology as a unifying framework for the Andreev versus Majorana states controversy, Commun. Phys. 2, 1 (2019).

[23] J. Ávila, E. Prada, P. San-Jose, and R. Aguado, Majorana oscillations and parity crossings in semiconductor nanowire-based transmon qubits, Phys. Rev. Res. 2, 033493 (2020).

[24] J. C. E. Saldaña, A. Vekris, L. Pavešič, P. Krogstrup, R. Žitko, K. Grove-Rasmussen, and J. Nygård, Bias 
asymmetric subgap states mimicking Majorana signatures, arXiv (2021), 2101.10794.

[25] M. T. Deng, S. Vaitiekènas, E. B. Hansen, J. Danon, M. Leijnse, K. Flensberg, J. Nygård, P. Krogstrup, and C. M. Marcus, Majorana bound state in a coupled quantum-dot hybrid-nanowire system, Science 354, 1557 (2016).

[26] J. Kondo, Resistance Minimum in Dilute Magnetic Alloys, Prog. Theor. Phys. 32, 37 (1964).

[27] A. C. Hewson, The Kondo Problem to Heavy Fermions, Cambridge Studies in Magnetism (Cambridge University Press, 1993).

[28] H. v. Löhneysen, A. Rosch, M. Vojta, and P. Wölfle, Fermi-liquid instabilities at magnetic quantum phase transitions, Rev. Mod. Phys. 79, 1015 (2007).

[29] Q. Si, R. Yu, and E. Abrahams, High-temperature superconductivity in iron pnictides and chalcogenides, Nat. Rev. Mater. 1, 1 (2016).

[30] S. Paschen and Q. Si, Quantum phases driven by strong correlations, Nat. Rev. Phys. 3, 9 (2021).

[31] M. Dzero, J. Xia, V. Galitski, and P. Coleman, Topological Kondo Insulators, Annu. Rev. Condens. Matter Phys. 7, 249 (2016).

[32] H.-H. Lai, S. E. Grefe, S. Paschen, and Q. Si, Weyl-Kondo semimetal in heavy-fermion systems, Proc. Natl. Acad. Sci. U.S.A. 115, 93 (2018).

[33] M. Cheng, M. Becker, B. Bauer, and R. M. Lutchyn, Interplay between Kondo and Majorana Interactions in Quantum Dots, Phys. Rev. X 4, 031051 (2014).

[34] J. F. Silva, L. G. G. V. D. da Silva, and E. Vernek, Robustness of the Kondo effect in a quantum dot coupled to Majorana zero modes, Phys. Rev. B 101, 075428 (2020).

[35] I. J. van Beek and B. Braunecker, Non-Kondo many-body physics in a Majorana-based Kondo type system, Phys. Rev. B 94, 115416 (2016).

[36] B. Béri and N. R. Cooper, Topological Kondo Effect with Majorana Fermions, Phys. Rev. Lett. 109, 156803 (2012).

[37] L. Herviou, K. Le Hur, and C. Mora, Many-terminal Majorana island: From topological to multichannel Kondo model, Phys. Rev. B 94, 235102 (2016).

[38] M. Kornjača, V. L. Quito, and R. Flint, Mobile Majorana zero-modes in two-channel Kondo insulators, arXiv (2021), 2104.11173.

[39] M. Lee, J. S. Lim, and R. López, Kondo effect in a quantum dot side-coupled to a topological superconductor, Phys. Rev. B 87, 241402(R) (2013).

[40] D. A. Ruiz-Tijerina, E. Vernek, L. G. G. V. Dias da Silva, and J. C. Egues, Interaction effects on a Majorana zero mode leaking into a quantum dot, Phys. Rev. B 91, 115435 (2015).

[41] I. Weymann and K. P. Wójcik, Transport properties of a hybrid Majorana wire-quantum dot system with ferromagnetic contacts, Phys. Rev. B 95, 155427 (2017).

[42] K. Flensberg, Tunneling characteristics of a chain of Majorana bound states, Phys. Rev. B 82, 180516(R) (2010).

[43] E. Vernek, P. H. Penteado, A. C. Seridonio, and J. C. Egues, Subtle leakage of a Majorana mode into a quantum dot, Phys. Rev. B 89, 165314 (2014).

[44] I. Weymann, K. P. Wójcik, and P. Majek, MajoranaKondo interplay in T-shaped double quantum dots, Phys. Rev. B 101, 235404 (2020).

[45] M. Pustilnik and L. I. Glazman, Kondo Effect in Real Quantum Dots, Phys. Rev. Lett. 87, 216601 (2001).

[46] P. S. Cornaglia and D. R. Grempel, Strongly correlated regimes in a double quantum dot device, Phys. Rev. B 71, 075305 (2005).

[47] S. Sasaki, H. Tamura, T. Akazaki, and T. Fujisawa, Fano-Kondo Interplay in a Side-Coupled Double Quantum Dot, Phys. Rev. Lett. 103, 266806 (2009).

[48] K. P. Wójcik and I. Weymann, Two-stage Kondo effect in T-shaped double quantum dots with ferromagnetic leads, Phys. Rev. B 91, 134422 (2015).

[49] X. Guo, Q. Zhu, L. Zhou, W. Yu, W. Lu, and W. Liang, Evolution and universality of two-stage Kondo effect in single manganese phthalocyanine molecule transistors, Nat. Commun. 12, 1 (2021).

[50] R. López, M. Lee, L. Serra, and J. S. Lim, Thermoelectrical detection of Majorana states, Phys. Rev. B 89, 205418 (2014).

[51] I. Weymann, Spin Seebeck effect in quantum dot sidecoupled to topological superconductor, J. Phys.: Condens. Matter 29, 095301 (2017).

[52] T. A. Costi and V. Zlatić, Thermoelectric transport through strongly correlated quantum dots, Phys. Rev. B 81, 235127 (2010).

[53] B. Dutta, D. Majidi, A. García Corral, P. A. Erdman, S. Florens, T. A. Costi, H. Courtois, and C. B. Winkelmann, Direct probe of the seebeck coefficient in a kondo-correlated single-quantumdot transistor, Nano Letters 19, 506 (2019), https://doi.org/10.1021/acs.nanolett.8b04398.

[54] A. Svilans, M. Josefsson, A. M. Burke, S. Fahlvik, C. Thelander, H. Linke, and M. Leijnse, Thermoelectric characterization of the kondo resonance in nanowire quantum dots, Phys. Rev. Lett. 121, 206801 (2018).

[55] G. E. W. Bauer, E. Saitoh, and B. J. van Wees, Spin caloritronics, Nat. Mater. 11, 391 (2012).

[56] W.-J. Gong, S.-F. Zhang, Z.-C. Li, G. Yi, and Y.-S. Zheng, Detection of a Majorana fermion zero mode by a T-shaped quantum-dot structure, Phys. Rev. B 89, 245413 (2014).

[57] J. P. Ramos-Andrade, O. Ávalos Ovando, P. A. Orellana, and S. E. Ulloa, Thermoelectric transport through Majorana bound states and violation of Wiedemann-Franz law, Phys. Rev. B 94, 155436 (2016).

[58] F. Buccheri, A. Nava, R. Egger, P. Sodano, and D. Giuliano, Violation of the Wiedemann-Franz law in the Topological Kondo model, arXiv (2021), 2108.04156.

[59] C.-Y. Hou, K. Shtengel, and G. Refael, Thermopower and Mott formula for a Majorana edge state, Phys. Rev. B 88, 075304 (2013).

[60] M. Leijnse, Thermoelectric signatures of a Majorana bound state coupled to a quantum dot, New J. Phys. 16, 015029 (2014).

[61] S. Valentini, R. Fazio, V. Giovannetti, and F. Taddei, Thermopower of three-terminal topological superconducting systems, Phys. Rev. B 91, 045430 (2015).

[62] S. Smirnov, Universal Majorana thermoelectric noise, Phys. Rev. B 97, 165434 (2018).

[63] X.-Q. Wang, S.-F. Zhang, Y. Han, G.-Y. Yi, and W.-J. Gong, Efficient enhancement of the thermoelectric effect due to the Majorana zero modes coupled to one quantumdot system, Phys. Rev. B 99, 195424 (2019).

[64] S. Smirnov, Dual Majorana universality in thermally induced nonequilibrium, Phys. Rev. B 101, 125417 (2020).

[65] K. P. Wójcik and I. Weymann, Thermopower of strongly correlated T-shaped double quantum dots, Phys. Rev. B 
93, 085428 (2016).

[66] D. Sherman, J. S. Yodh, S. M. Albrecht, J. Nygård, P. Krogstrup, and C. M. Marcus, Normal, superconducting and topological regimes of hybrid double quantum dots, Nat. Nanotechnol. 12, 212 (2017).

[67] J. I. Väyrynen, D. I. Pikulin, and R. M. Lutchyn, Majorana signatures in charge transport through a topological superconducting double-island system, arXiv (2020), 2010.05963.

[68] D. J. Carrad, M. Bjergfelt, T. Kanne, M. Aagesen, F. Krizek, E. M. Fiordaliso, E. Johnson, J. Nygård, and T. S. Jespersen, Shadow Epitaxy for In Situ Growth of Generic Semiconductor/Superconductor Hybrids, Adv. Mater. 32, 1908411 (2020).

[69] S. Heedt, M. Quintero-Pérez, F. Borsoi, A. Fursina, N. van Loo, G. P. Mazur, M. P. Nowak, M. Ammerlaan, K. Li, S. Korneychuk, J. Shen, M. A. Y. van de Poll, G. Badawy, S. Gazibegovic, K. van Hoogdalem, E. P. A. M. Bakkers, and L. P. Kouwenhoven, Shadow-wall lithography of ballistic superconductor-semiconductor quantum devices, arXiv (2020), 2007.14383.

[70] T. Kanne, M. Marnauza, D. Olsteins, D. J. Carrad, J. E. Sestoft, J. de Bruijckere, L. Zeng, E. Johnson, E. Olsson, K. Grove-Rasmussen, and J. Nygård, Epitaxial $\mathrm{Pb}$ on InAs nanowires, arXiv (2020), 2002.11641.

[71] F. Münning, O. Breunig, H. F. Legg, S. Roitsch, D. Fan, M. Rößler, A. Rosch, and Y. Ando, Quantum confinement of the Dirac surface states in topological-insulator nanowires, Nat. Commun. 12, 1 (2021).

[72] M. M. Desjardins, L. C. Contamin, M. R. Delbecq, M. C. Dartiailh, L. E. Bruhat, T. Cubaynes, J. J. Viennot, F. Mallet, S. Rohart, A. Thiaville, A. Cottet, and T. Kontos, Synthetic spin-orbit interaction for Majorana devices, Nat. Mater. 18, 1060 (2019).

[73] K. Delfanazari, L. Serra, P. Ma, R. K. Puddy, T. Yi, M. Cao, Y. Gul, I. Farrer, D. A. Ritchie, H. J. Joyce, M. J. Kelly, and C. G. Smith, Experimental evidence for topological phases in the magnetoconductance of 2DEGbased hybrid junctions, arXiv (2020), 2007.02057.

[74] D. E. Liu and H. U. Baranger, Detecting a Majoranafermion zero mode using a quantum dot, Phys. Rev. B 84, 201308(R) (2011).

[75] S. M. Albrecht, A. P. Higginbotham, M. Madsen, F. Kuemmeth, T. S. Jespersen, J. Nygård, P. Krogstrup, and C. M. Marcus, Exponential protection of zero modes in Majorana islands, Nature 531, 206 (2016).

[76] G. Benenti, G. Casati, K. Saito, and R. S. Whitney, Fundamental aspects of steady-state conversion of heat to work at the nanoscale, Phys. Rep. 694, 1 (2017).

[77] R. Barnard, Thermoelectricity in Metals and Alloys (Taylor \& Francis, 1972).

[78] L. G. G. V. Dias da Silva, E. Vernek, K. Ingersent, N. Sandler, and S. E. Ulloa, Spin-polarized conductance in double quantum dots: Interplay of Kondo, Zeeman, and interference effects, Phys. Rev. B 87, 205313 (2013).

[79] K. P. Wójcik and I. Weymann, Perfect spin polariza- tion in T-shaped double quantum dots due to the spindependent Fano effect, Phys. Rev. B 90, 115308 (2014).

[80] K. G. Wilson, The renormalization group: Critical phenomena and the Kondo problem, Rev. Mod. Phys. 47, 773 (1975).

[81] R. Bulla, T. A. Costi, and T. Pruschke, Numerical renormalization group method for quantum impurity systems, Rev. Mod. Phys. 80, 395 (2008).

[82] O. Legeza, C. P. Moca, A. I. Tóth, I. Weymann, and G. Zaránd, Manual for the Flexible DMNRG code, arXiv:0809.3143v1 (2008), (the open access Flexible DM-NRG Budapest code is available at http://www.phy.bme.hu/ dmnrg/.

[83] F. B. Anders and A. Schiller, Real-time dynamics in quantum-impurity systems: A time-dependent numerical renormalization-group approach, Phys. Rev. Lett. 95, 196801 (2005).

[84] G. D. Mahan and J. O. Sofo, The best thermoelectric, Proc. Natl. Acad. Sci. U.S.A. 93, 7436 (1996).

[85] F. Haldane, Scaling theory of the asymmetric Anderson model, Phys. Rev. Lett. 40, 416 (1978).

[86] R. Franz and G. Wiedemann, Ueber die WärmeLeitungsfähigkeit der Metalle, Ann. Phys. 165, 497 (1853).

[87] D. Giuliano, A. Nava, R. Egger, P. Sodano, and F. Buccheri, Multi-particle scattering and breakdown of the Wiedemann-Franz law at a junction of $\mathrm{N}$ interacting quantum wires, arXiv (2021), 2108.04149.

[88] I. Weymann, Finite-temperature spintronic transport through Kondo quantum dots: Numerical renormalization group study, Phys. Rev. B 83, 113306 (2011).

[89] T. A. Costi, Kondo Effect in a Magnetic Field and the Magnetoresistivity of Kondo Alloys, Phys. Rev. Lett. 85, 1504 (2000).

[90] J. Martinek, M. Sindel, L. Borda, J. Barnaś, R. Bulla, J. König, G. Schön, S. Maekawa, and J. von Delft, Gatecontrolled spin splitting in quantum dots with ferromagnetic leads in the Kondo regime, Phys. Rev. B 72, 121302 (2005).

[91] S. B. Tooski, A. Ramšak, B. R. Bułka, and R. Žitko, Effect of assisted hopping on thermopower in an interacting quantum dot, New J. Phys. 16, 055001 (2014).

[92] I. Weymann and J. Barnaś, Spin thermoelectric effects in Kondo quantum dots coupled to ferromagnetic leads, Phys. Rev. B 88, 085313 (2013).

[93] R. Świrkowicz, M. Wierzbicki, and J. Barnaś, Thermoelectric effects in transport through quantum dots attached to ferromagnetic leads with noncollinear magnetic moments, Phys. Rev. B 80, 195409 (2009).

[94] M. Misiorny and J. Barnaś, Effect of magnetic anisotropy on spin-dependent thermoelectric effects in nanoscopic systems, Phys. Rev. B 91, 155426 (2015).

[95] R. Wiesendanger, Spin mapping at the nanoscale and atomic scale, Rev. Mod. Phys. 81, 1495 (2009).

[96] I. Žutić, J. Fabian, and S. Das Sarma, Spintronics: Fundamentals and applications, Rev. Mod. Phys. 76, 323 (2004). 